\title{
Cardiovascular disease and COPD: dangerous liaisons?
}

\author{
Klaus F. Rabe ${ }^{1,2}$, John R. Hurst ${ }^{3}$ and Samy Suissa ${ }^{4,5}$
}

Affiliations: ${ }^{1}$ Dept of Medicine, University of Kiel, Kiel, Germany. ${ }^{2}$ Lung Clinic Großhansdorf, Airway Research Center North (ARCN), Großhansdorf, Germany. ${ }^{3}$ Centre for Inflammation and Tissue Repair, Division of Medicine, University College London, London, UK. ${ }^{4}$ Centre for Clinical Epidemiology, Lady Davis Institute, Jewish General Hospital, Montreal, QC, Canada. ${ }^{5}$ Dept of Epidemiology and Biostatistics, McGill University, Montreal, QC, Canada.

Correspondence: Klaus F. Rabe, LungenClinic Großhansdorf, Airway Research Center North, Woehrendamm 80, Großhansdorf 22927, Germany. E-mail: k.f.rabedlungenclinic.de

@ERSpublications

CVD and COPD are often comorbid. Their pathophysiology and treatment may affect each other and health outcomes. http://ow.ly/v18p30lxmms

Cite this article as: Rabe KF, Hurst JR, Suissa S. Cardiovascular disease and COPD: dangerous liaisons? Eur Respir Rev 2018; 27: 180057 [https://doi.org/10.1183/16000617.0057-2018].

ABSTRACT Chronic obstructive pulmonary disease (COPD) and cardiovascular disease (CVD) frequently occur together and their coexistence is associated with worse outcomes than either condition alone. Pathophysiological links between COPD and CVD include lung hyperinflation, systemic inflammation and COPD exacerbations. COPD treatments may produce beneficial cardiovascular (CV) effects, such as longacting bronchodilators, which are associated with improvements in arterial stiffness, pulmonary vasoconstriction, and cardiac function. However, data are limited regarding whether these translate into benefits in $\mathrm{CV}$ outcomes. Some studies have suggested that treatment with long-acting $\beta_{2}$-agonists and longacting muscarinic antagonists leads to an increase in the risk of CV events, particularly at treatment initiation, although the safety profile of these agents with prolonged use appears reassuring. Some CV medications may have a beneficial impact on COPD outcomes, but there have been concerns about $\beta$-blocker use leading to bronchospasm in COPD, which may result in patients not receiving guideline-recommended treatment. However, there are few data suggesting harm with these agents and patients should not be denied $\beta$-blockers if required. Clearer recommendations are necessary regarding the identification and management of comorbid CVD in patients with COPD in order to facilitate early intervention and appropriate treatment.

\section{Introduction}

Chronic obstructive pulmonary disease (COPD) is a complex respiratory disorder characterised by chronic airflow limitation and an increased inflammatory response of the lung [1]. COPD is associated with many comorbidities [2,3] (figure 1) and can be one of multiple chronic or acute diseases and medical conditions present within one person [4]. In particular, cardiovascular disease (CVD) and COPD share similar risk factors such as ageing, history of cigarette smoking (or other exposures) and a sedentary lifestyle, and frequently coexist $[2,3,5]$.

Historically, many terms and definitions have been inconsistently used to describe the co-existence of diseases [6]. Recently, it was proposed that the term "index disease" should be used to describe the main condition of interest and "comorbidity" for any other medical conditions present at diagnosis of the index disease or later [6]. By contrast, "multimorbidity" is defined simply as the co-existence of two or more chronic diseases $[6,7]$.

This article has been amended according to the erratum published in the December 2018 issue of the European Respiratory Review.

Provenance: Submitted article, peer reviewed.

Received: June 192018 | Accepted after revision: Aug 202018

Copyright OERS 2018. ERR articles are open access and distributed under the terms of the Creative Commons Attribution Non-Commercial Licence 4.0. 


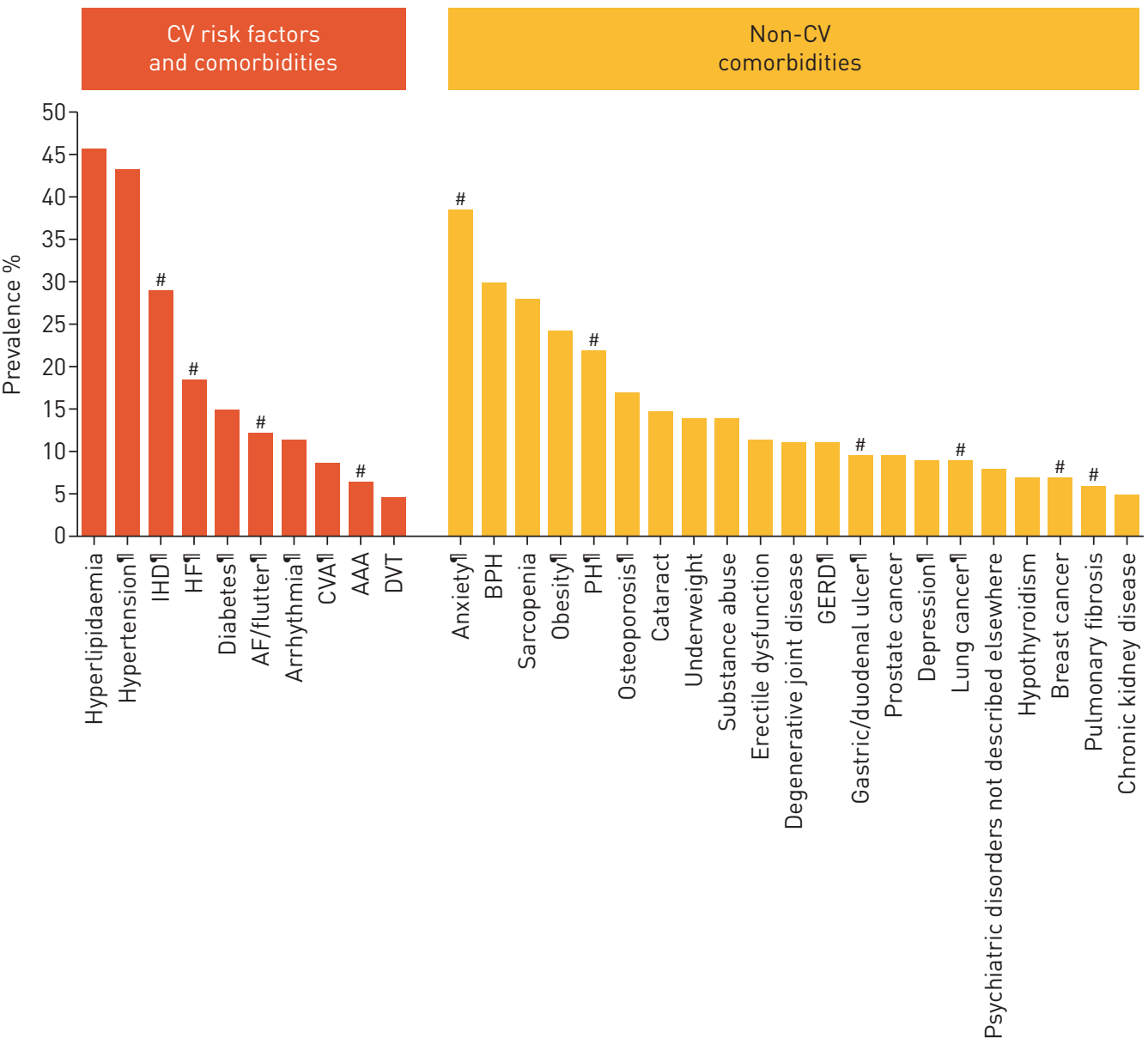

FIGURE 1 Prevalence of comorbidities in pooled studies of patients with chronic obstructive pulmonary disease (COPD). Cardiovascular (CV) conditions and comorbidities are highlighted as orange bars and other comorbidities as yellow bars. Data are pooled from numerous studies and comorbidities with a prevalence (calculated as a weighted average based on study sample size) $>5 \%$ are shown. IHD: ischaemic heart disease; HF: heart failure; AF: atrial fibrillation; CVA: cerebrovascular accident; AAA: abdominal aortic aneurysm; DVT: deep vein thrombosis; BPH: benign prostatic hypertrophy; $\mathrm{PH}$ : pulmonary hypertension; GERD: gastrooesophageal reflux disease. ${ }^{\#}$ : comorbidities with a significant increase in mortality risk compared to patients with COPD without the comorbidity; ${ }^{\text {I: }}$ comorbidities with a significantly increased prevalence in patients with COPD compared with the general population. Reproduced and modified with permission from [3].

Patients with comorbid COPD and CVD experience high rates of morbidity, including worse quality of life, dyspnoea and exercise tolerance [8], and a higher risk of hospitalisation for COPD and for CVD [9]. In addition, the presence of CVD or cardiovascular (CV) conditions (such as heart failure (HF), ischaemic heart disease (IHD), diabetes or atrial fibrillation (AF)) increases the risk of frequent exacerbations [10] and mortality $[2,8,11]$ (figure 2). Further, COPD exacerbations and lung function decline are associated with increased CV risk and mortality $[12,13]$. To minimise the risk of poor outcomes, it is therefore important to ensure that patients with comorbid COPD and CVD are managed effectively. However, drug therapies for COPD could have both beneficial and potential adverse effects on CVD and vice versa.

In the current review we provide a brief overview of key pathophysiological mechanisms, which may help explain comorbid COPD and CVD and inform the rationale for treatment. In the light of the plethora of recent literature considering the CV risks of pharmacotherapy in patients with COPD [14-18] we then provide a synthesis of the research from clinical trials and observational studies to establish the evidence for the main classes of drugs used to treat COPD and CVD. Finally, reflecting on our findings, we make some recommendations for the management of COPD patients with CVD.

\section{Pathophysiological links between COPD and CVD}

The mechanisms which underlie the association between COPD and CVD are not well understood but several processes are thought to be important and may interact with each other [19, 20]. These include 


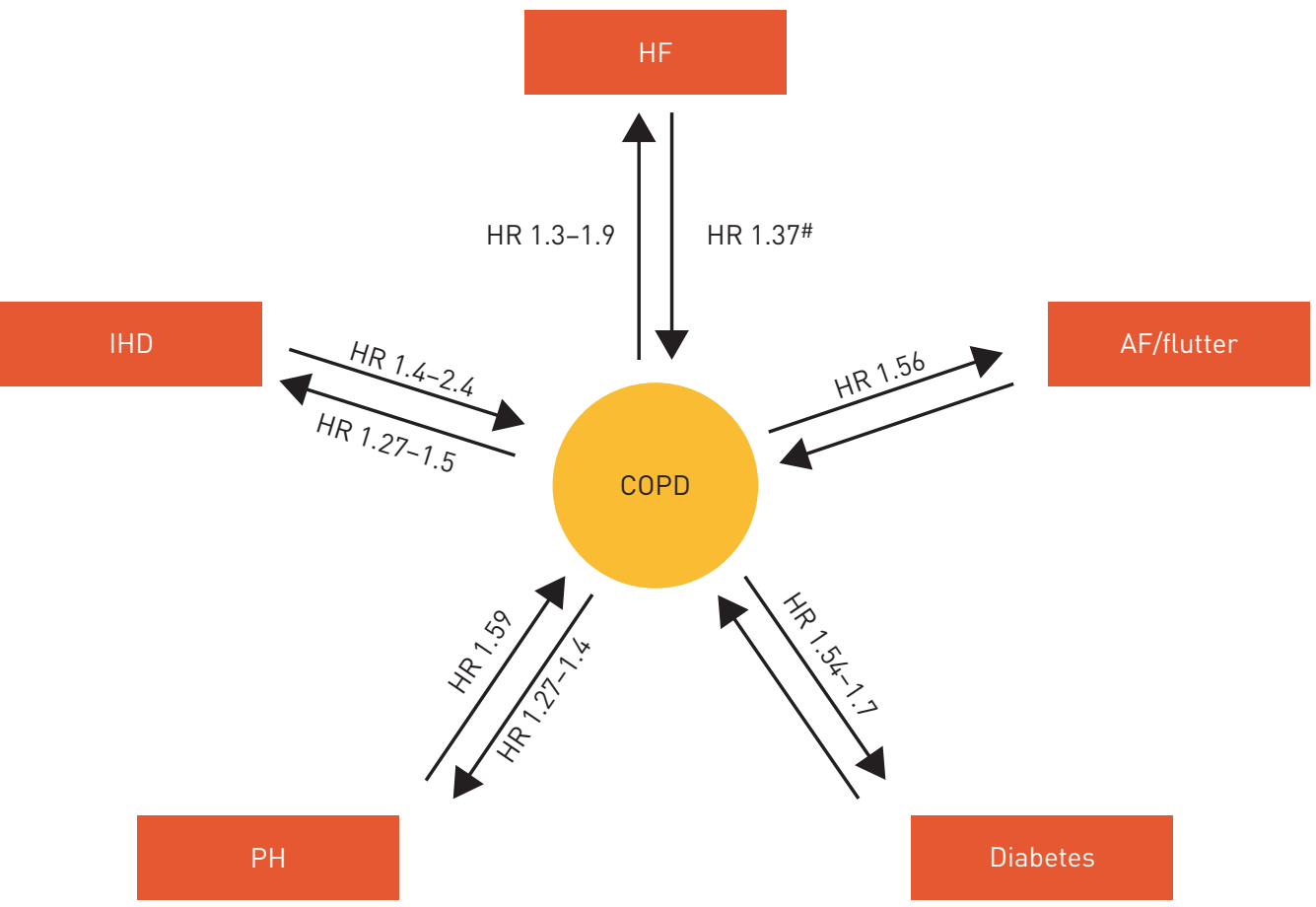

FIGURE 2 Association between cardiovascular disease, cardiovascular risk factors and chronic obstructive pulmonary disease (COPD) on mortality. Arrows and associated values indicate the impact of each comorbidity on the mortality risk of patients with COPD versus those patients with COPD alone. Where available, the reverse is also shown (the impact of COPD on the mortality risk of patients with each comorbidity versus those with the comorbidity alone). AF: atrial fibrillation; HF: heart failure; HR: hazard ratio; IHD: ischaemic heart disease; PH: pulmonary hypertension. ${ }^{\#}$ : HR was not significant after adjustment for confounding risk factors. Reproduced and modified with permission from [3].

lung hyperinflation, hypoxaemia, pulmonary hypertension $(\mathrm{PH})$, systemic inflammation and oxidative stress, exacerbations, shared risk factors and shared genetics (figure 3), as well as COPD phenotype.

Hyperinflation, characterised by abnormally elevated residual gas in the lungs following spontaneous exhalation [21], is a major driver of COPD burden and mortality [1]. It is the cardinal pathophysiological mechanism affecting the mechanics of breathing and can be either static (resulting from destruction of the lung parenchyma and subsequent loss of lung elastic recoil) or dynamic (occurring when a patient inhales

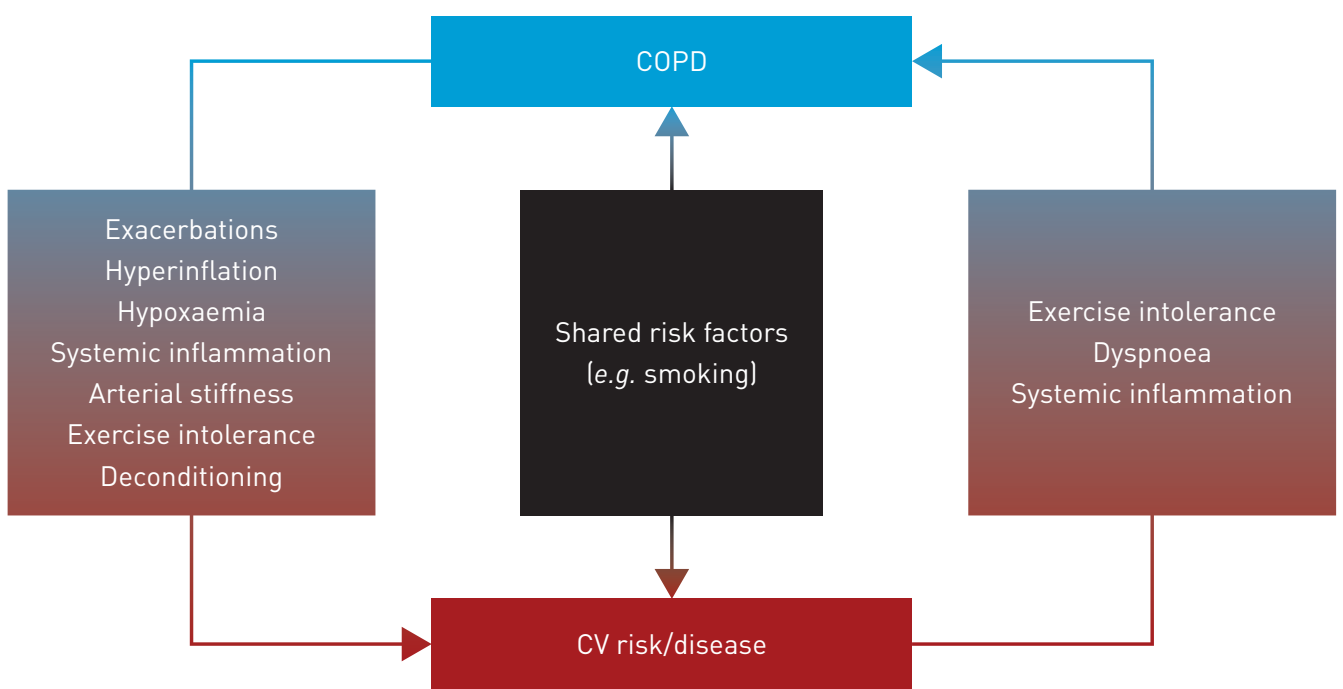

FIGURE 3 Potential interactions between chronic obstructive pulmonary disease (COPD), cardiovascular (CV) risk and cardiovascular disease. 
before exhaling fully, trapping air with each additional breath) [21]. Hyperinflation significantly reduces the efficiency of the respiratory muscles [22] and is increasingly recognised as a major cause of dyspnoea (shortness of breath) [23].

Abnormal lung function, including hyperinflation, is also thought to compromise cardiac function through various means [24]. Notably, airflow limitation caused by lung hyperinflation may cause increased pressures in the cardiopulmonary system, right-ventricular dysfunction, impaired left-ventricular filling and reduced cardiac output (QT) [25-29]. Emphysema is associated with static hyperinflation [30] and hyperinflation may therefore be a key risk factor for CVD in patients with emphysema-predominant COPD. In addition, progressive airflow limitation and emphysema in COPD lead to a ventilation/ perfusion mismatch that is a key contributor to the development of hypoxaemia, which can be further exacerbated by exercise and sleep disordered breathing [31]. Hypoxaemia in patients with COPD can lead to pulmonary vasoconstriction and vascular remodelling, resulting in right-ventricular diastolic dysfunction [32]. Indeed, $\mathrm{PH}$, common in patients with severe COPD, can lead to right HF, which is in turn associated with left HF $[33,34]$. In addition, altered cardiac repolarisation in patients with COPD may be related to hypoxaemia and could increase the risk of ventricular arrhythmias and sudden cardiac death [35].

Chronic or intermittent hypoxia may also increase systemic inflammation, which is known to play a role in the pathogenesis of CVD $[36,37]$ and has been linked to the development of arterial stiffness which has a strong predictive value for $\mathrm{CV}$ events [38]. Markers of pulmonary inflammation (such as surfactant protein D) and of systemic inflammation (such as C-reactive protein (CRP)) are elevated in patients with stable COPD [8, 39-41], while patients with COPD and CVD have higher blood concentrations of inflammatory markers, such as fibrinogen, interleukin (IL)-6 and IL-8, than those without CVD [8]. Moreover, coronary artery calcification scores (a marker of coronary atherosclerosis) correlate with markers of systemic and pulmonary inflammation, such as IL-6, IL-8, surfactant protein D and peripheral blood neutrophil count, in patients with COPD [42]. These findings suggest that COPD is either a systemic inflammatory state or that inflammatory processes spill over from the lungs into the systemic circulation [43], contributing to the development of CVD.

The occurrence of COPD exacerbations has been shown to increase the risk of subsequent CV events in patients with CVD or CV risk factors [44]. This may be related to lung inflammation which is heightened during COPD exacerbations [24]. Furthermore, lower respiratory tract infections, a common cause of COPD exacerbation, are associated with increased inflammatory markers such as fibrinogen and IL-6 which are linked with thrombosis and CV events $[12,45]$. High levels of inflammation and oxidative stress that occur during and after an exacerbation may reduce circulating CD34+ cells (which are involved in vascular repair) and increase platelet activation and arterial stiffness [45-49]. Of note, the sudden increase in airway resistance increases that occurs during an exacerbation further limits expiratory flow and lung emptying. Consequently, patients experiencing an exacerbation tend to adopt a rapid, shallow breathing pattern, resulting in a vicious cycle of diminishing lung emptying time and increasing dynamic hyperinflation [22].

The source of CVD risk may be associated with the dominance of either chronic bronchitis or emphysema within the COPD disease profile. The presence of emphysema may reflect accelerated ageing of the lung, which may also result in HF due to downregulation of anti-ageing molecules such as sirtuins [50], while the presence of chronic bronchitis could reflect a distinct inflammatory subtype of COPD requiring specific anti-inflammatory interventions. The genetic background of chronic bronchitis and emphysema also differ, which may influence the processes underlying the association between COPD and CVD [51].

The physiological effects of CVD, for example HF-associated dyspnoea (due to pulmonary oedema) and reduced exercise capacity (due to reduced QT and impaired perfusive and diffusive oxygen transport) $[52,53]$ may add to the effects of COPD caused by "pulmonary" mechanisms (dyspnoea due to hyperinflation and resulting exercise avoidance and deconditioning [1]). In HF, cardiomegaly may be involved in causing a restrictive lung pattern and reduced alveolar volume [54], with alveolar gas diffusion progressively worsening due to reduction in the lung tissue participating in gas exchange [55]. Ventilatory response to exercise is greater than normal for a given metabolic rate, due to an increase in physiological dead space to tidal volume ratio (driven by high ventilation/perfusion mismatching, increased carbon dioxide production relative to oxygen uptake from lactate buffering and a decrease in partial pressure of carbon dioxide) [56]. The clinical consequences are illustrated in a study in which patients with COPD and IHD had significantly worse health status, dyspnoea and exercise capacity than those without IHD; additionally, although exacerbations were not more frequent, recovery time was longer in those with IHD [57]. CVD is also a leading cause of hospitalisation and mortality in patients with COPD [9]. 
Although the causal or consequential role of associated systemic inflammation is still not clear in COPD $[58,59]$, as described earlier, there are a number of mechanisms by which COPD-associated systemic inflammation could contribute to CVD and low-grade systemic inflammation in patients with airflow obstruction has been associated with increased risk of cardiac injury [40]. Systemic inflammation is also a feature of CVD, likely playing a role in its development and progression [60]. While the effect of CVD-associated systemic inflammation on COPD is unknown, a study in COPD patients with and without hepatitis C found a steeper decline in lung function with hepatitis C versus controls [61], suggesting an effect on the lungs due to systemic inflammation. Furthermore, risk factors of systemic and vascular inflammation, such as visceral obesity, diabetes and inactivity are also associated with reduced pulmonary function, airway hyper-reactivity and eventually COPD [58], supporting the idea that systemic inflammation associated with CVD may affect COPD.

In summary, many of the pathophysiological mechanisms underlying COPD may increase the risk of CVD and vice versa; however, more studies are needed to provide evidence that the mechanisms uncovered are relevant to the clinical manifestations of these diseases when they occur together.

\section{Pharmacological management of COPD and CVD}

With the close association between COPD and CVD, it is possible that treatments for one condition may influence the other and appropriate treatment of all conditions is therefore essential. Improvements in survival following hospital discharge for acute COPD exacerbation have been attributed to better management of COPD and associated comorbidities, including the use of CV therapies such as angiotensin-converting enzyme inhibitors (ACEIs), angiotensin receptor blockers (ARBs), $\beta$-blockers and statins [62]. However, the treatment of $\mathrm{CV}$ conditions in patients with COPD is associated with therapeutic challenges, most notably the co-administration of (selective) $\beta_{1}$-blockers and $\beta_{2}$-agonists. $\beta$-blockers are widely prescribed in the treatment of CVD and $\beta$-agonists represent a cornerstone of COPD treatment; however, the two have opposing pharmacological actions and physicians may be concerned that treatment of one condition may worsen the other [63].

A number of randomised controlled trials (RCTs) and observational studies provide insight into the effect of COPD treatments on CVD and vice versa, although both study types are subject to limitations. For instance, results from observational studies may be affected by lack of blinding of study treatments and confounding bias [64]. Also, observational studies are often reliant upon patient databases, which may lack accuracy (for instance, the definition of COPD and the presence of comorbidities such as asthma) [64-66]. In addition, a number of observational analyses discussed below rely upon prescription data, which may not accurately reflect actual medication intake $[65,66]$. Conversely, RCTs are associated with limitations of their own, such as their limited size and duration of exposure, as well as a lack of generalisability to the general COPD population due to stringent inclusion criteria and trial setting [64]. Moreover, RCTs may introduce bias due to the deleterious effect of treatment withdrawal at randomisation, the inclusion of a run-in period, the use of untreated placebo groups and by truncating follow-up at treatment discontinuation [67-69].

\section{Effects of COPD treatments on CV risk}

Among COPD patients, CV causes are the second most common cause of death (following pulmonary causes) [70]. In this section, we discuss the effects of different classes of COPD treatment on CV risk and mortality, followed by the effects of different classes of CV treatment on COPD. Details from key studies $[27,65,66,71-124]$ are summarised in tables 1 and 2.

\section{Bronchodilators}

There is conflicting evidence regarding the CV safety of bronchodilators. An increased CV risk has been observed with bronchodilators in some studies [72, 76, 84, 125], whereas others found no evidence of increased risk or even some evidence of CV risk reduction $[89,126]$.

\section{$\boldsymbol{\beta}_{2}$-agonists}

While a systematic review of long-acting $\beta_{2}$-agonists (LABAs), in combination with inhaled corticosteroids (ICS), concluded that ICS/LABA products may have a good CV safety profile in asthma patients [127], evidence for the CV safety of LABAs in COPD is less definitive. LABAs have been associated with an increased risk of $\mathrm{CV}$ events, possibly due to stimulation of sympathetic drive [72, 84], which may also increase the risk of arrhythmias and myocardial ischaemia in patients with CVD (particularly HF). In clinical trials, $\beta_{2}$-agonists have been shown to increase heart rate, reduce potassium concentrations and increase the risk of CV events versus placebo in patients with obstructive airways disease [72]. Furthermore, studies of Canadian healthcare databases indicate that new use of LABAs is associated with an elevated rate of cardiac arrhythmia in patients with COPD [82, 83]. In addition, a recent analysis of 


\begin{tabular}{|c|c|c|c|c|c|c|c|}
\hline Author/study & Design and participants & Duration & Population & Treatment(s) & Primary outcome & Key results & Other findings \\
\hline \multicolumn{8}{|l|}{$\beta_{2}$-agonists } \\
\hline $\begin{array}{l}\text { SuISSA } \\
\text { (2003) [71] }\end{array}$ & $\begin{array}{l}\text { Population-based, nested } \\
\text { case-control } \\
(n=12090)\end{array}$ & $\begin{array}{l}\text { Follow-up to } \\
1999\end{array}$ & $\begin{array}{l}\text { Newly diagnosed patients } \\
\text { with COPD aged } \\
>55 \text { years (identified } \\
\text { from the Saskatchewan } \\
\text { Health Services } \\
\text { databases, 1980-1997) }\end{array}$ & SABAs & $\begin{array}{l}\text { Cases of acute } \mathrm{MI} \\
\text { occurring during } \\
\text { cohort follow-up }\end{array}$ & $\begin{array}{l}1127 \text { evaluable patients } \\
\text { identified with fatal or } \\
\text { non-fatal acute MI } \\
\text { SABA use not associated } \\
\text { with increased risk of } \\
\text { acute } \mathrm{Ml} \text { (rate ratio for } \\
\text { any use: } 1.06,95 \% \mathrm{Cl} \\
0.92-1.23 \text { ) }\end{array}$ & $\begin{array}{l}\text { No significant increase in } \\
\text { risk when analysis is } \\
\text { restricted to patients } \\
\text { with } \mathrm{CV} \text { risk factors }\end{array}$ \\
\hline $\begin{array}{l}\text { SALPETER } \\
(2004)[72]\end{array}$ & $\begin{array}{l}\text { Meta-analysis of } \\
\text { randomised and } \\
\text { placebo-controlled } \\
\text { trials } \\
13 \text { RCTs of single-dose } \\
\text { treatment ( } n=232 \text { ) } \\
20 \text { trials of longer } \\
\text { treatment duration } \\
\text { (n=6623) }\end{array}$ & $\begin{array}{l}\text { Mean of } \\
4.7 \text { months } \\
\text { (range: } \\
3 \text { days to } \\
1 \text { year) }\end{array}$ & $\begin{array}{l}\text { Patients with obstructive } \\
\text { airway disease (COPD } \\
\text { or asthma) } \\
\text { Mean age } 56.6 \text { years } \\
\text { (single-dose) and } \\
52.2 \text { years (longer } \\
\text { treatment studies) }\end{array}$ & $\begin{array}{l}\beta_{2} \text {-agonists versus } \\
\text { placebo }\end{array}$ & $\begin{array}{l}\text { Short-term effect on } \\
\text { heart rate and } \\
\text { potassium } \\
\text { concentrations } \\
\text { Long-term effect on } \\
\text { adverse CV events }\end{array}$ & $\begin{array}{l}\text { Single-dose } \beta_{2} \text {-agonist } \\
\text { versus placebo } \\
\text { increased heart rate by } \\
9.12 \mathrm{bpm} \text { and reduced } \\
\text { potassium concentration } \\
\text { by } 0.36 \mathrm{mmol} \cdot \mathrm{L}^{-1} \\
\text { Longer } \beta_{2} \text {-agonist } \\
\text { treatment significantly } \\
\text { increased risk for CV } \\
\text { events (relative risk } \\
2.54,95 \% \mathrm{Cl} 1.59-4.05 \text { ) }\end{array}$ & $\begin{array}{l}\text { The relative risk for sinus } \\
\text { tachycardia was } 3.06 \\
(95 \% \mathrm{Cl} 1.70-5.50) \\
\text { For all other events, } \\
\text { relative risk was } 1.66 \\
(95 \% \mathrm{Cl} 0.76-3.6)\end{array}$ \\
\hline $\begin{array}{l}\text { CAzZoLA } \\
\text { (2007) [27] }\end{array}$ & $\begin{array}{l}\text { Randomised, double } \\
\text { blind, double dummy } \\
(\mathrm{n}=20)\end{array}$ & & $\begin{array}{l}\text { COPD and sPAP } \\
>20 \mathrm{mmHg} \text { (rest) }\end{array}$ & $\begin{array}{l}\text { Salmeterol } 50 \mu \mathrm{g} \\
\text { Formoterol } 12 \mu \mathrm{g}\end{array}$ & $\begin{array}{l}\text { Acute haemodynamic } \\
\text { response }\end{array}$ & $\begin{array}{l}\text { Mean sPAP significantly } \\
\text { decreased versus } \\
\text { baseline at } 15,30 \text { and } \\
60 \text { min post-inhalation } \\
(p<0.05)\end{array}$ & $\begin{array}{l}\text { No correlation between } \\
\text { maximum increase in } \\
\text { FEV1 and maximum } \\
\text { decrease in SPAP after } \\
\text { inhalation of } \\
\text { salmeterol or } \\
\text { formoterol }\end{array}$ \\
\hline $\begin{array}{l}\text { SAnTUS } \\
\text { (2015) [73] }\end{array}$ & $\begin{array}{l}\text { Randomised, double } \\
\text { blind, placebo } \\
\text { controlled, crossover } \\
(n=40)\end{array}$ & & $\begin{array}{l}\text { COPD (FEV } 1 \leqslant 70 \% \text { pred; } \\
\text { RV } \geqslant 135 \% \text { pred) } \\
\text { Absence of } \mathrm{CV} \\
\text { comorbidities } \\
\text { Age } 50-85 \text { years }\end{array}$ & $\begin{array}{l}\text { Indacaterol } 150 \mu \mathrm{g} \\
\text { versus placebo } \\
\text { (1:1) }\end{array}$ & $\begin{array}{l}\text { Effect of reduction of } \\
\text { right-ventricular } \\
\text { and FRC on right } \\
\text { heart systolic/ } \\
\text { diastolic functional } \\
\text { indices }\end{array}$ & $\begin{array}{l}\text { Significant improvements } \\
\text { in right-ventricular } \\
\text { compliance/cardiac } \\
\text { performance with } \\
\text { indacaterol versus } \\
\text { placebo ( } \leqslant \leqslant 0.05 \text { after } \\
180 \text { min treatment) as } \\
\text { follows: } \\
\text { 1) TAPSE: } 0.41 \mathrm{~mm} \\
\text { versus } 0.02 \mathrm{~mm} \\
\text { 2) DT-TR: } 11.9 \mathrm{~ms} \\
\text { versus } 3.8 \mathrm{~ms} \\
\text { 3) } \leqslant \text { Heart rate: }-2 \mathrm{bpm} \\
\text { versus } 0.6 \mathrm{bpm}\end{array}$ & $\begin{array}{l}\text { Indacaterol associated } \\
\text { with significant } \\
(p<0.05) \text { increases } \\
\text { versus placebo in FEV1, } \\
\text { VC and IC }\end{array}$ \\
\hline
\end{tabular}




\begin{tabular}{|c|c|c|c|c|c|c|c|}
\hline Author/study & Design and participants & Duration & Population & Treatment(s) & Primary outcome & Key results & Other findings \\
\hline \multicolumn{8}{|l|}{$\begin{array}{l}\text { Muscarinic } \\
\text { antagonists }\end{array}$} \\
\hline $\begin{array}{l}\text { KESTEN } \\
2006[74]\end{array}$ & $\begin{array}{l}\text { Pooled safety analysis of } \\
19 \text { randomised, double } \\
\text { blind, placebo } \\
\text { controlled trials } \\
\text { (including two asthma } \\
\text { trials) (n=7819) }\end{array}$ & & $\begin{array}{l}\text { COPD diagnosis with } \\
\text { airflow limitation } \\
\text { Age } \geqslant 40 \text { years } \\
\text { Smoking history } \geqslant 10 \\
\text { pack-years }\end{array}$ & $\begin{array}{l}\text { Tiotropium } 18 \mu \mathrm{g} \\
\text { once daily } \\
\text { (n=4435) } \\
\text { Placebo (n=3384) }\end{array}$ & $\begin{array}{l}\text { AEs occurring during } \\
\text { the study }\end{array}$ & $\begin{array}{l}\text { Tiotropium not associated } \\
\text { with increased risk of } \\
\text { serious cardiac events } \\
\text { versus placebo. Data as } \\
\text { follows: } \\
\text { 1) CV mortality (relative } \\
\text { risk } 0.57,95 \% \mathrm{Cl} \\
0.26-1.26 \text { ) } \\
\text { 2) Cardiac arrest } \\
\text { (relative risk 0.90, } \\
95 \% \mathrm{Cl} 0.26-3.15 \text { ) } \\
\text { 3) Ml (relative risk } 0.74, \\
\text { 95\% Cl 0.26-2.07) }\end{array}$ & $\begin{array}{l}\text { Relative risk of } \\
\text { tachycardia with } \\
\text { tiotropium versus } \\
\text { placebo: } 1.68 \text { (95\% Cl } \\
\text { 0.69-4.11) }\end{array}$ \\
\hline $\begin{array}{l}\text { TRAVERS } \\
\text { (2007) [75] }\end{array}$ & $\begin{array}{l}\text { Randomised, double } \\
\text { blind, placebo } \\
\text { controlled, crossover } \\
\text { (n=18) }\end{array}$ & $\begin{array}{l}\text { 7-10 day } \\
\text { treatment } \\
\text { period }+ \\
35 \text { day } \\
\text { washout } \\
\text { period }\end{array}$ & $\begin{array}{l}\text { COPD }(F E V 1 \leqslant 65 \% \\
\text { pred; FRC } \geqslant 120 \% \\
\text { pred; modified } \\
\text { baseline dyspnoea } \\
\text { index score } \leqslant 6 \text { ) }\end{array}$ & $\begin{array}{l}\text { Tiotropium } 18 \mu \mathrm{g} \\
\text { once daily versus } \\
\text { placebo }\end{array}$ & $\begin{array}{l}\text { Effect of tiotropium on } \\
\text { CV response to } \\
\text { exercise }\end{array}$ & $\begin{array}{l}\text { Tiotropium improved } \\
\text { cardiac function during } \\
\text { exercise versus placebo: } \\
\text { 1) Significantly reduced } \\
\text { heart rate (105 bpm } \\
\text { versus } 112 \mathrm{bpm}, \mathrm{p}<0.05 \text { ) } \\
\text { 2) Higher } \mathrm{O}_{2} \text { pulse } \\
\text { (10.9 } \mathrm{mL} \cdot \text { beat }^{-1} \text { versus } \\
10.1 \mathrm{~mL} \cdot \text { beat }^{-1}, p<0.05 \text { ) } \\
\text { 3) Lower SBP } \\
\text { (148 } \mathrm{mmHg} \text { versus } \\
156 \mathrm{mmHg}, p<0.05 \text { ) }\end{array}$ & $\begin{array}{l}\text { Tiotropium significantly } \\
\text { improved measures of } \\
\text { dynamic hyperinflation } \\
\text { during exercise: IRV } \\
\text { was significantly } \\
\text { greater with tiotropium } \\
\text { versus placebo }(0.60 \mathrm{~L} \\
\text { versus } 0.44 \mathrm{~L}, \mathrm{p}<0.05)\end{array}$ \\
\hline $\begin{array}{l}\text { SINGH } \\
\text { (2008) [76] }\end{array}$ & $\begin{array}{l}\text { Systematic review and } \\
\text { meta-analysis ( } 17 \\
\text { RCTs; } 12 \text { on tiotropium, } \\
5 \text { on ipratropium) (n=14 } \\
783 \text { ) }\end{array}$ & & COPD of any severity & $\begin{array}{l}\text { Tiotropium or } \\
\text { ipratropium } \\
\text { (n=7472) versus } \\
\text { control (placebo/ } \\
\text { active control) } \\
\text { (n=7311) }\end{array}$ & $\begin{array}{l}\text { Composite of } \\
\text { non-fatal MI, } \\
\text { non-fatal stroke and } \\
\text { CV death }\end{array}$ & $\begin{array}{l}\text { Tiotropium or ipratropium } \\
\text { significantly increased } \\
\text { risk of } \mathrm{CV} \text { death, } \mathrm{MI} \text { or } \\
\text { stroke versus control } \\
(1.8 \% \text { versus } 1.2 \% \text {; } \\
\text { relative risk } 1.58 \\
(95 \% \mathrm{Cl} 1.21-2.06) \\
\mathrm{p}<0.001)\end{array}$ & $\begin{array}{l}\text { Tiotropium or ipratropium } \\
\text { did not significantly } \\
\text { increase risk of } \\
\text { all-cause mortality } \\
\text { versus control ( } 2.0 \% \\
\text { versus } 1.6 \% \text {; relative } \\
\text { risk } 1.26(95 \% \mathrm{Cl} \\
0.99-1.61), \mathrm{p}=0.06)\end{array}$ \\
\hline $\begin{array}{l}\text { RodRIG0 } \\
\text { (2009) [77] }\end{array}$ & $\begin{array}{l}\text { Systematic review and } \\
\text { meta-analysis (19 } \\
\text { RCTs) ( } n=18111)\end{array}$ & $\begin{array}{l}7 \text { trials (28- } \\
48 \text { months) } \\
12 \text { trials } \\
\text { (8 weeks- } \\
6 \text { months) }\end{array}$ & $\begin{array}{l}\text { Patients with COPD } \\
\text { (average baseline FEV1: } \\
41 \% \text { pred normal) } \\
\text { Mean age } 64.8 \text { years }\end{array}$ & $\begin{array}{l}\text { Tiotropium versus } \\
\text { placebo }\left(n=15^{\#}\right) \text {, } \\
\text { SFC }\left(n=2^{\#}\right) \text {, } \\
\text { salmeterol }\left(n=1^{\#}\right) \text {, } \\
\text { salmeterol/ } \\
\text { placebo }\left(n=1^{\#}\right)\end{array}$ & $\begin{array}{l}\text { Composite of MACE, } \\
\text { CV mortality and } \\
\text { non-fatal MI or } \\
\text { stroke }\end{array}$ & $\begin{array}{l}\text { No difference in incidence } \\
\text { of MACE versus control } \\
\text { groups (relative risk } \\
0.96,95 \% \mathrm{Cl} 0.82-1.12 \text { ) }\end{array}$ & $\begin{array}{l}\text { Compared with control } \\
\text { groups, tiotropium did } \\
\text { not significantly } \\
\text { increase risk of: } \\
\text { 1) CV death (relative } \\
\text { risk } 0.93,95 \% \mathrm{Cl} \\
0.73-1.20 \text { ) } \\
\text { 2) Non-fatal MI } \\
\text { (relative risk 0.84, } \\
95 \% \mathrm{Cl} 0.64-1.09 \text { ) } \\
\text { 3) Non-fatal stroke } \\
\text { (relative risk 1.04, } \\
95 \% \mathrm{Cl} 0.78-1.39 \text { ) }\end{array}$ \\
\hline
\end{tabular}




\begin{tabular}{|c|c|c|c|c|c|c|c|}
\hline Author/study & Design and participants & Duration & Population & Treatment(s) & Primary outcome & Key results & Other findings \\
\hline $\begin{array}{l}\text { CELLI }(2009, \\
\text { UPLIFT) } \\
{[78]}\end{array}$ & $\begin{array}{l}\text { Multicentre, randomised, } \\
\text { double blind, placebo- } \\
\text { controlled, parallel } \\
\text { group ( } n=5993 \text { ) } \\
\text { Mortality analysis }\end{array}$ & 4 years & $\begin{array}{l}\text { Patients aged >40 years } \\
\text { with COPD } \\
\text { (post-bronchodilator } \\
\text { FEV } 1 \leqslant 70 \% \text { pred normal; } \\
\text { FEV } 1 / \text { FV } \leqslant 70 \% \text { ) }\end{array}$ & $\begin{array}{l}\text { Tiotropium ( } n=2987) \\
\text { Placebo }(n=3006)\end{array}$ & $\begin{array}{l}\text { Effect of tiotropium on } \\
\text { survival }\end{array}$ & $\begin{array}{l}\text { Reduced risk of cardiac } \\
\text { mortality with } \\
\text { tiotropium versus } \\
\text { placebo (HR 0.86, } 95 \% \\
\text { Cl } 0.75-0.99 \text { ) }\end{array}$ & $\begin{array}{l}\text { Reduced risk of all-cause } \\
\text { mortality for } \\
\text { tiotropium versus } \\
\text { placebo (HR 0.84 (95\% } \\
\text { Cl 0.73-0.97), } p=0.016 \text { ) }\end{array}$ \\
\hline $\begin{array}{l}\text { NoJIRI } 2012 \\
\text { [79] }\end{array}$ & $\begin{array}{l}\text { Single-centre, } \\
\text { prospective, pilot }(n=21)\end{array}$ & 12 weeks & $\begin{array}{l}\text { Patients with COPD } \\
\left(\mathrm{FEV}_{1} / \mathrm{FVC}<0.70\right) \text { and } \\
\text { prior }(\geqslant 1 \text { year) } \\
\text { pulmonary resection } \\
\text { for lung cancer } \\
\text { ECOG status } 1 \\
>20 \text { pack-year smoking } \\
\text { history }\end{array}$ & $\begin{array}{l}\text { Tiotropium } 18 \mu \mathrm{g} \\
\text { once daily }\end{array}$ & $\begin{array}{l}\text { Pulmonary function } \\
\text { and left-ventricular } \\
\text { diastolic dysfunction } \\
\text { [E/e' ratio }]\end{array}$ & $\begin{array}{l}\text { Significant improvements } \\
\text { after tiotropium (versus } \\
\text { before tiotropium) in: } \\
\text { 1) FEV1 }(1.84 \mathrm{~L} \text { versus } \\
\text { 1.60 L, } p<0.001) \\
\text { 2) E/e' ratio ( } 7.59 \text { versus } \\
\text { 8.97, } p<0.001)\end{array}$ & $\begin{array}{l}\text { No significant differences } \\
\text { before/after tiotropium } \\
\text { in BP, heart rate, FVC, } \\
\text { left-ventricular mass } \\
\text { and LVEF } \\
\text { Significant } \\
\text { improvement in PASP } \\
\text { versus before } \\
\text { tiotropium }(33.0 \mathrm{mmHg} \\
\text { versus } 38.5 \mathrm{mmHg} \text {, } \\
\text { p<0.01) }\end{array}$ \\
\hline $\begin{array}{l}\text { PEPIN } \\
(2014)[80]\end{array}$ & $\begin{array}{l}\text { Multicentre, randomised, } \\
\text { double dummy, parallel } \\
\text { group, blinded ( } n=257)\end{array}$ & 12 weeks & $\begin{array}{l}\text { COPD patients aged } \\
\geqslant 40 \text { years } \\
\text { Smoking history } \\
\geqslant 10 \text { pack-years } \\
\text { Post-bronchodilator FEV } 1 \\
\leqslant 70 \% \text { pred normal } \\
\text { FEV } 1 / F V C \leqslant 0.70 \\
\text { aPWV } \geqslant 11 \mathrm{~m} \cdot \mathrm{s}^{-1}\end{array}$ & $\begin{array}{l}\text { Tiotropium } 18 \mu \mathrm{g} \\
\text { once daily ( } \mathrm{n}=130) \\
\text { versus fluticasone } \\
\text { furoate/vilanterol } \\
\text { combination } \\
100 \mu \mathrm{g} / 25 \mu \mathrm{g} \text { once } \\
\text { daily }(\mathrm{n}=127)\end{array}$ & $\begin{array}{l}\text { Change from baseline } \\
\text { in arterial stiffness } \\
(\mathrm{aPWV})^{+} \text {at } 12 \text { weeks }\end{array}$ & $\begin{array}{l}\text { At } 12 \text { weeks there was a } \\
\text { comparable reduction } \\
\text { from baseline in aPWV } \\
\text { with tiotropium } \\
\left(-1.118 \mathrm{~m} \cdot \mathrm{s}^{-1}\right) \text { and } \\
\text { fluticasone furoate/ } \\
\text { vilanterol combination } \\
\left(-0.859 \mathrm{~m} \cdot \mathrm{s}^{-1}, \mathrm{p}=\mathrm{NS}\right)\end{array}$ & $\begin{array}{l}\text { No significant differences } \\
\text { between tiotropium } \\
\text { and fluticasone } \\
\text { furoate/vilanterol } \\
\text { combination for } \\
\text { change from baseline } \\
\text { in trough } \mathrm{FEV} 1(0.080 \mathrm{~L} \\
\text { versus } 0.117 \mathrm{~L}) \text { or IC } \\
(0.019 \mathrm{~L} \text { versus } 0.089 \mathrm{~L})\end{array}$ \\
\hline $\begin{array}{l}\text { SuISSA } \\
\text { (2017) [65] }\end{array}$ & $\begin{array}{l}\text { UK observational, } \\
\text { population-based prior } \\
\text { ICS-matched cohort } \\
\text { analysis (primary care, } \\
\text { CPRD) (n=115397, base } \\
\text { cohort (new users of a } \\
\text { long-acting } \\
\text { bronchodilator); } n=70 \\
550, \text { sub-cohort (linked } \\
\text { to HES database)) }\end{array}$ & 1 year & $\begin{array}{l}\text { New users of long-acting } \\
\text { bronchodilators (LABA } \\
\text { or tiotropium) for COPD } \\
\text { Aged }>55 \text { years } \\
\geqslant 2 \text { years medical history } \\
\text { First LABA/tiotropium } \\
\text { prescription on/after } \\
\text { September } 25,2003\end{array}$ & $\begin{array}{l}\text { Full cohort } \\
\text { (matched by } \\
\text { propensity score): } \\
\text { tiotropium ( } n=26 \\
\text { 442), LABA ( } n=26 \\
\text { 442) } \\
\text { HES sub-cohort: } \\
\text { tiotropium ( } n=15 \\
\text { 427), LABA ( } n=15 \\
\text { 427) }\end{array}$ & $\begin{array}{l}\text { Incidence of acute MI, } \\
\text { stroke, HF (full } \\
\text { cohort) and incidence } \\
\text { of arrhythmia and } \\
\text { pneumonia (HES } \\
\text { sub-cohort) following } \\
1 \text { year of treatment } \\
\text { with tiotropium } \\
\text { versus LABA }\end{array}$ & $\begin{array}{l}\text { No difference between } \\
\text { tiotropium versus LABA } \\
\text { in CV events, as follows: } \\
\text { 1) Acute MI: HR 1.10 } \\
\text { (95\% CI 0.88-1.38) } \\
\text { 2) Stroke: HR 1.02 } \\
\text { (95\% Cl 0.78-1.34) } \\
\text { 3) Heart failure: HR } 0.90 \\
\text { (95\% Cl 0.79-1.02) } \\
\text { 4) Arrhythmia: HR 0.81 } \\
\text { (95\% Cl 0.60-1.09) }\end{array}$ & $\begin{array}{l}\text { Risk of pneumonia } \\
\text { significantly reduced } \\
\text { with tiotropium versus } \\
\text { LABA (HR } 0.81,95 \% \mathrm{Cl} \\
\text { 0.72-0.92) }\end{array}$ \\
\hline \multicolumn{8}{|c|}{$\begin{array}{l}\beta_{2} \text {-agonists } \\
\text { and muscarinic } \\
\text { antagonists }\end{array}$} \\
\hline $\begin{array}{l}\text { BERTON } \\
\text { (2010) [81] }\end{array}$ & $\begin{array}{l}\text { Double-blind, placebo- } \\
\text { controlled, crossover } \\
\text { (n=12) }\end{array}$ & & $\begin{array}{l}\text { Moderate-to-severe COPD } \\
\quad(\mathrm{FEV} 1 / \mathrm{FVC}<0.7 ; \\
\text { post-bronchodilator } \\
\text { FEV } 1<60 \% \text { pred) } \\
\text { Resting } \mathrm{PaO}_{2}>60 \mathrm{mmHg}\end{array}$ & $\begin{array}{l}\text { Salbutamol } 120 \mu \mathrm{g}+ \\
\text { ipratropium } \\
20 \mu \mathrm{g} / \text { actuation } \\
\text { versus placebo }\end{array}$ & $\begin{array}{l}\text { Key determinants of } \\
\mathrm{O}_{2} \text { delivery and } \\
\text { uptake during } \\
\text { high-intensity, } \\
\text { constant work rate } \\
\text { cycling exercise }\end{array}$ & $\begin{array}{l}\text { Compared with placebo, } \\
\text { bronchodilators } \\
\text { accelerated central } \\
\text { haemodynamic response } \\
\text { at exercise onset, as } \\
\text { follows: } \\
\text { 1) } \mathrm{t}_{1 / 2} \mathrm{QT}: 75.9 \pm 10.3 \mathrm{~s} \\
\text { versus } 58.9 \pm 18.9 \mathrm{~s} \text { ( } \mathrm{p}=0.02 \text { ) }\end{array}$ & $\begin{array}{l}\text { Bronchodilators led to } \\
\text { lung deflation and } \\
\text { increased exercise } \\
\text { tolerance versus } \\
\text { placebo }(454 \pm 131 \mathrm{~s} \\
\text { versus } 321 \pm 140 \mathrm{~s} ; \\
p<0.05)\end{array}$ \\
\hline
\end{tabular}




\begin{tabular}{|c|c|c|c|c|c|c|c|}
\hline Author/study & Design and participants & Duration & Population & Treatment(s) & Primary outcome & Key results & Other findings \\
\hline & & & & & & $\begin{array}{l}\text { 2) } \mathrm{t}_{1 / 2} \text { heart rate: } \\
78.2 \pm 13.0 \mathrm{~s} \text { versus } \\
62.5 \pm 15.5 \mathrm{~s} \text { ( } \mathrm{p}=0.03) \\
\text { 3) } \mathrm{t}_{1 / 2} \mathrm{sV}: 51.0 \pm 8.1 \mathrm{~s} \\
\text { versus } 40.6 \pm 10.3 \mathrm{~s}(\mathrm{p}=0.02)\end{array}$ & \\
\hline $\begin{array}{l}\text { WILCHESKY } \\
\text { (2012-part } \\
\text { 1) [82] }\end{array}$ & $\begin{array}{l}\text { Retrospective, cohort } \\
\text { (healthcare databases, } \\
\text { Province of } \\
\text { Saskatchewan, Canada) } \\
\text { (n=6018) }\end{array}$ & & $\begin{array}{l}\text { COPD patients aged } \\
\geqslant 55 \text { years } \\
\text { Newly treated COPD } \\
\text { (three or more } \\
\text { prescriptions for a } \\
\text { bronchodilator, on two } \\
\text { different dates, within } \\
\text { any 1-year period) }\end{array}$ & $\begin{array}{l}\text { LABA } \\
\text { SABA } \\
\text { Methylxanthines } \\
\text { Ipratropium } \\
\text { bromide }\end{array}$ & $\begin{array}{c}\text { Rate of cardiac } \\
\text { arrhythmias }\end{array}$ & $\begin{array}{l}\text { Rate of arrhythmia } \\
\text { increased with new use } \\
\text { of: } \\
\text { 1) ipratropium (relative } \\
\text { risk } 2.4,95 \% \mathrm{Cl} 1.4-4.0 \text { ) } \\
\text { 2) LABA (relative risk 4.5, } \\
\text { 95\% } \mathrm{Cl} 1.4-14.4 \text { ) } \\
\text { Rate of arrhythmia was not } \\
\text { increased with new use } \\
\text { of: } \\
\text { 1) SABA (relative risk } 0.9 \text {, } \\
\text { 95\% CI 0.5-1.6) } \\
\text { 2) Methylxanthines } \\
\text { (relative risk 1.6, 95\% Cl } \\
\text { 0.7-3.7) }\end{array}$ & \\
\hline $\begin{array}{l}\text { WILCHESKY } \\
\text { (2012-part } \\
\text { 2) [83] }\end{array}$ & $\begin{array}{l}\text { Retrospective, cohort } \\
\text { (healthcare databases, } \\
\text { Quebec, Canada) ( } \mathrm{n}=76 \\
\text { 661) }\end{array}$ & & $\begin{array}{l}\text { COPD patients aged } \\
\geqslant 67 \text { years } \\
\text { Newly treated COPD } \\
\text { (three or more } \\
\text { prescriptions for a } \\
\text { bronchodilator, on two } \\
\text { different dates between } \\
\text { January 01, 1990 and } \\
\text { December } 31,2002 \text { ) }\end{array}$ & $\begin{array}{l}\text { LABA } \\
\text { SABA } \\
\text { Methylxanthines } \\
\text { Ipratropium } \\
\text { bromide }\end{array}$ & $\begin{array}{c}\text { Rate of cardiac } \\
\text { arrhythmias }\end{array}$ & $\begin{array}{l}\text { Rate of arrhythmia } \\
\text { increased with new use } \\
\text { of: } \\
\text { 1) SABA (relative risk } \\
\text { 1.27, 95\% Cl 1.03-1.57) } \\
\text { 2) LABA (relative risk } \\
\text { 1.47, } 95 \% \mathrm{Cl} 1.01-2.15 \text { ) } \\
\text { Rate of arrhythmia slightly } \\
\text { (not significantly) } \\
\text { increased with new use } \\
\text { of: } \\
\text { 1) ipratropium bromide } \\
\text { (relative risk 1.23, } \\
\text { 95\% Cl 0.95-1.57) } \\
\text { 2) Methylxanthines } \\
\text { (relative risk 1.28, } \\
\text { 95\% Cl 0.93-1.77) }\end{array}$ & \\
\hline $\begin{array}{l}\text { GERSHON } \\
\text { (2013) [84] }\end{array}$ & $\begin{array}{l}\text { Population-based, nested } \\
\text { case-control analysis } \\
\text { of a retrospective study } \\
\text { (n=191005) }\end{array}$ & & $\begin{array}{l}\text { COPD patients aged } \\
\geqslant 66 \text { years } \\
\text { Receiving treatment } \\
\text { (September 2003- } \\
\text { March 2009) } \\
53532 \text { had a CV event } \\
26628 \text { matched to } \\
\text { control }^{\S}\end{array}$ & $\begin{array}{l}\text { LABA } \\
\text { LAMA }\end{array}$ & $\begin{array}{r}\text { Hospitalisation or ED } \\
\text { visit for a CV event }\end{array}$ & $\begin{array}{l}\text { New users of LABAs and } \\
\text { LAMAs more likely } \\
\text { versus non-users to } \\
\text { have a CV-related } \\
\text { hospitalisation/ED visit } \\
\text { LABAs: OR } 1.31(95 \% \mathrm{Cl} \\
\text { 1.12-1.52), } \mathrm{p}<0.001 \\
\text { LAMAs: OR } 1.14(95 \% \mathrm{Cl} \\
1.01-1.28), \mathrm{p}=0.03\end{array}$ & $\begin{array}{l}\text { No significant difference } \\
\text { in CV events between } \\
\text { LABAs and LAMAs } \\
\text { (OR } 1.15(95 \% \mathrm{Cl} \\
0.95-1.38), \mathrm{p}=0.16)\end{array}$ \\
\hline
\end{tabular}




\begin{tabular}{|c|c|c|c|c|c|c|c|}
\hline Author/study & Design and participants & Duration & Population & Treatment(s) & Primary outcome & Key results & Other findings \\
\hline $\begin{array}{l}\text { WANG } \\
{[2018)} \\
{[123]}\end{array}$ & $\begin{array}{l}\text { Nested case-control } \\
\text { study (healthcare } \\
\text { database, Taiwan) } \\
\text { (n=284229) }\end{array}$ & & $\begin{array}{l}\text { COPD patients aged } \\
\geqslant 40 \text { years } \\
\text { LABA-LAMA naïve } \\
37719 \text { had a CV event } \\
\text { Matched with } 146139 \\
\text { randomly selected } \\
\text { controls }\end{array}$ & $\begin{array}{l}\text { LABA } \\
\text { LAMA }\end{array}$ & $\begin{array}{l}\text { Inpatient or ED visit } \\
\text { for CAD, HF, } \\
\text { ischaemic stroke or } \\
\text { arrhythmia }\end{array}$ & $\begin{array}{l}\text { New LABA and LAMA use } \\
\text { were associated with an } \\
\text { increased risk of a CV } \\
\text { event within } 30 \text { days (OR } \\
1.50 \text { ( } 95 \% \text { Cl } 1.35-1.67), \\
\text { p<0.001 and OR } 1.52 \text { (95\% } \\
\text { Cl 1.28-1.80), p<0.001, } \\
\text { respectively). The risk was } \\
\text { absent, or even reduced, } \\
\text { with prevalent use }\end{array}$ & $\begin{array}{l}\text { No difference in risk was } \\
\text { observed between } \\
\text { individual LABA } \\
\text { agents, LAMA dosage } \\
\text { forms, or concomitant } \\
\text { COPD regimens }\end{array}$ \\
\hline \multicolumn{8}{|c|}{$\begin{array}{l}\text { LABA/LAMA } \\
\text { combinations }\end{array}$} \\
\hline $\begin{array}{l}\text { SuISSA } \\
\text { (2017) [66] }\end{array}$ & $\begin{array}{l}\text { UK observational, } \\
\text { population-based cohort } \\
\text { analysis (primary care, } \\
\text { CPRD) ( } n=463899 \text {, base } \\
\text { cohort) } \\
31174 \text { patients on } \\
\text { combined bronchodilator } \\
\text { therapy matched to } 31 \\
174 \text { patients on } \\
\text { bronchodilator } \\
\text { monotherapy }\end{array}$ & 1 year & $\begin{array}{l}\text { New users of long-acting } \\
\text { bronchodilators (LABA } \\
\text { or tiotropium) for COPD } \\
\text { Aged }>55 \text { years } \\
\geqslant 2 \text { years medical history } \\
\text { First LABA/tiotropium } \\
\text { prescription on/after } \\
\text { September } 25,2002\end{array}$ & $\begin{array}{l}\text { LABA/tiotropium } \\
\text { initiation + second } \\
\text { long-acting } \\
\text { bronchodilator } \\
\text { (n=31 174) } \\
\text { Bronchodilator } \\
\text { monotherapy } \\
\text { (n=31 174) }\end{array}$ & $\begin{array}{l}\text { Incidence of acute MI, } \\
\text { stroke, HF and } \\
\text { arrhythmia } \\
\text { following } 1 \text { year of } \\
\text { treatment }\end{array}$ & $\begin{array}{l}\text { Combination of two } \\
\text { long-acting } \\
\text { bronchodilators was not } \\
\text { associated with } \\
\text { increased risk of: } \\
\text { 1) Acute MI (HR 1.12, } \\
\text { 95\% Cl 0.92-1.37) } \\
\text { 2) Stroke (HR 0.87, } \\
\text { 95\% Cl 0.69-1.10) } \\
\text { 3) Arrhythmia (HR } 1.05 \text {, } \\
\text { 95\% Cl 0.81-1.36) }\end{array}$ & $\begin{array}{l}\text { Two long-acting } \\
\text { bronchodilators in } \\
\text { combination were } \\
\text { associated with } \\
\text { increased risk of HF } \\
\text { (HR 1.16, 95\% Cl } \\
1.03-1.30 \text { ) }\end{array}$ \\
\hline $\begin{array}{l}\text { SAMP (2017) } \\
\text { [85] }\end{array}$ & $\begin{array}{l}\text { Retrospective, } \\
\text { observational cohort } \\
\text { lusing health insurance } \\
\text { claims data) ( } n=19078 \\
\text { matched patients) }\end{array}$ & & $\begin{array}{l}\text { Patients with COPD } \\
\text { initiating LABA/LAMA } \\
\text { or ICS/LABA }\end{array}$ & $\begin{array}{l}\text { LABA/LAMA } \\
\text { (n=3844) } \\
\text { ICS/LABA ( } n=15 \\
234)\end{array}$ & $\begin{array}{l}\text { CCV outcomes: } \\
\text { hospitalisations for } \\
\text { ACS, HF, cardiac } \\
\text { dysrhythmia, stroke, } \\
\text { or TIA }\end{array}$ & $\begin{array}{l}\text { LABA/LAMA treatment was } \\
\text { associated with fewer CV } \\
\text { events versus ICS/LABA } \\
\text { treatment (HR 0.794, } \\
95 \% \mathrm{Cl} 0.623-0.997 \text { ) }\end{array}$ & $\begin{array}{l}\text { No difference between } \\
\text { treatments in } \\
\text { cerebrovascular events } \\
\text { (HR 1.166, 95\% Cl } \\
0.653-1.959 \text { ) }\end{array}$ \\
\hline $\begin{array}{l}\text { HOHLFELD } \\
\text { (2017) [86] }\end{array}$ & $\begin{array}{l}\text { Randomised, double- } \\
\text { blind, single centre, } \\
\text { placebo-controlled, two } \\
\text { period, crossover } \\
\text { (n=62) }\end{array}$ & & $\begin{array}{l}\text { Patients with COPD and } \\
\text { increased RV ( }>135 \% \\
\text { predicted) without CVD }\end{array}$ & $\begin{array}{l}\text { Indacaterol/ } \\
\text { glycopyrronium } \\
110 \mu \mathrm{g} / 50 \mu \mathrm{g} \text { once } \\
\text { daily } \\
\text { Placebo }\end{array}$ & $\begin{array}{l}\text { Change in LVEDV } \\
\text { measured with MRI } \\
\text { on day } 14\end{array}$ & $\begin{array}{l}\text { Compared with placebo, } \\
\text { indacaterol/ } \\
\text { glycopyrronium resulted } \\
\text { in a significant increase } \\
\text { in LVEDV }(10.27 \mathrm{~mL}, \\
p<0.0001) \text { and } Q T \\
\left(0.337 \mathrm{~L} \cdot \mathrm{min}^{-1},\right. \\
p=0.0032)\end{array}$ & $\begin{array}{l}\text { Compared with placebo, } \\
\text { indacaterol/ } \\
\text { glycopyrronium was } \\
\text { associated with: } \\
\text { 1) Improved lung } \\
\text { function (peak FEV1 } \\
\text { increased by } 0.42 \mathrm{~L} \text {, } \\
\text { p<0.0001) } \\
\text { 2) Reduced lung } \\
\text { hyperinflation (RV } \\
\text {-0.75 L, p<0.0001) }\end{array}$ \\
\hline \multicolumn{8}{|c|}{$\begin{array}{l}\text { ICS and ICS/LABA } \\
\text { combinations }\end{array}$} \\
\hline $\begin{array}{l}\text { HUIART } \\
\text { (2005) [87] }\end{array}$ & $\begin{array}{l}\text { Nested case-control } \\
\text { analysis (health } \\
\text { services databases, } \\
\text { Saskatchewan, Canada) } \\
\text { (n=5648) }\end{array}$ & $\begin{array}{l}\text { Follow-up } \\
\text { until first } \\
\text { MI }\end{array}$ & $\begin{array}{l}\text { Patients } \geqslant 55 \text { years with } \\
\text { new-onset COPD who } \\
\text { had not received any } \\
\text { bronchodilator, } \\
\text { anti-asthma drug or }\end{array}$ & ICS & $\begin{array}{l}\text { First fatal or non-fatal } \\
\text { acute MI }\end{array}$ & $\begin{array}{l}\text { Results based on } 371 \\
\text { cases with first acute MI } \\
\text { matched to } 1864 \\
\text { controls } \\
\text { Low-dose ICS } \\
\left(50-200 \mu \mathrm{g} \cdot \text { day }^{-1}\right)\end{array}$ & $\begin{array}{l}\text { Overall, current use of } \\
\text { ICS was not associated } \\
\text { with a significant } \\
\text { decrease in risk of } \\
\text { acute } \mathrm{MI} \text { (rate ratio } \\
0.82,95 \% \mathrm{Cl} 0.57-1.16 \text { ) }\end{array}$ \\
\hline
\end{tabular}




\begin{tabular}{|c|c|c|c|c|c|c|c|}
\hline Author/study & Design and participants & Duration & Population & Treatment(s) & Primary outcome & Key results & Other findings \\
\hline & & & $\begin{array}{l}\text { nasal/ICS in the } \\
\text { previous } 5 \text { years }\end{array}$ & & & $\begin{array}{l}\text { significantly reduced } \\
\text { risk of acute } \mathrm{MI} \text { by } 32 \% \\
\text { (rate ratio } 0.68,95 \% \mathrm{Cl} \\
0.47-0.99 \text { ) }\end{array}$ & \\
\hline $\begin{array}{l}\text { LOKE }(2010) \\
{[88]}\end{array}$ & $\begin{array}{l}\text { Systematic review of } 23 \\
\text { RCTs ( } \mathrm{n}=23396 \text { ) and } 12 \\
\text { observational studies }\end{array}$ & $\begin{array}{l}\text { RCTs } \\
\quad \geqslant 24 \text { weeks }\end{array}$ & $\begin{array}{l}\text { Patients with COPD of any } \\
\text { severity }\end{array}$ & $\begin{array}{l}\text { ICS versus placebo } \\
\text { or ICS/LABA } \\
\text { versus LABA }\end{array}$ & $\begin{array}{l}\text { Risk of fatal and } \\
\text { non-fatal MI and CV } \\
\text { death }\end{array}$ & $\begin{array}{l}\text { Findings from RCTs } \\
\text { indicated that ICS were } \\
\text { not associated with } \\
\text { significantly reduced } \\
\text { risk of } \mathrm{MI} \text { (relative risk } \\
0.95,95 \% \mathrm{Cl} 0.73-1.23 \text { ), } \\
\mathrm{CV} \text { death (relative risk } \\
1.02,95 \% \mathrm{Cl} 0.81-1.27 \text { ) } \\
\text { or mortality (relative } \\
\text { risk } 0.96,95 \% \mathrm{Cl} \\
0.86-1.07 \text { ) }\end{array}$ & $\begin{array}{l}\text { Findings from } \\
\text { observational studies } \\
\text { indicated that ICS use } \\
\text { was associated with a } \\
\text { significant reduction in } \\
\text { CV death (two studies: } \\
\text { relative risk } 0.79 \text { ( } 95 \% \\
\text { Cl } 0.72-0.86 \text { ), } \\
\text { p<0.0001) and } \\
\text { mortality (11 studies: } \\
\text { relative risk } 0.78 \text { ( } 95 \% \\
\text { Cl } 0.75-0.80 \text { ), } \\
\text { p<0.0001) }\end{array}$ \\
\hline $\begin{array}{l}\text { CALVERLEY } \\
\text { (2010, } \\
\text { TORCH) } \\
{[89]}\end{array}$ & $\begin{array}{l}\text { Multicentre, randomised, } \\
\text { double-blind, placebo- } \\
\text { controlled, parallel } \\
\text { group (post hoc } \\
\text { analysis) ( } \mathrm{n}=6184 \text { ) }\end{array}$ & 3 years & $\begin{array}{l}\text { Patients (current/former } \\
\text { smokers) with COPD } \\
\text { (pre-bronchodilator } \\
\text { FEV } 1<60 \% \text { pred and } \\
\text { FEV } 1 / F_{1} \text { FV } \leqslant 0.70 \text { ) } \\
\text { Aged } 40-80 \text { years }\end{array}$ & $\begin{array}{l}\text { Salmeterol/ } \\
\text { fluticasone } \\
\text { propionate } \\
\text { combination } \\
50 \mu \mathrm{g} / 500 \mu \mathrm{g} \\
\text { (n=1546) } \\
\text { Salmeterol } 50 \mu \mathrm{g} \\
\text { (n=1542) } \\
\text { Fluticasone } \\
\text { propionate } 500 \mu \mathrm{g} \\
\text { (n=1552) } \\
\text { Placebo (n=1544) } \\
\text { (all twice daily) }\end{array}$ & CV AEs and SAEs & $\begin{array}{l}\text { The probability of patients } \\
\text { having a CV AE by } \\
3 \text { years was lowest for } \\
\text { salmeterol/fluticasone } \\
\text { propionate combination } \\
(20.8 \%) \text { versus placebo } \\
(24.2 \%) \text {, salmeterol } \\
(22.7 \%) \text { and fluticasone } \\
\text { propionate }(24.3 \%)\end{array}$ & $\begin{array}{l}\text { Treatment with } \\
\text { salmeterol/fluticasone } \\
\text { propionate combination } \\
\text { was associated with a } \\
\text { significant reduction } \\
\text { versus placebo in } \\
\text { probability of a CV AE } \\
\text { by } 3 \text { years in patients } \\
\text { receiving CV therapy at } \\
\text { baseline (27.9\% versus } \\
33.5 \%, \text { respectively; } \\
\text { p }<0.05 \text { ) }\end{array}$ \\
\hline $\begin{array}{l}\text { DRANSFIELD } \\
\text { (2011) [90] }\end{array}$ & $\begin{array}{l}\text { Multicentre, randomised, } \\
\text { double-blind, placebo- } \\
\text { controlled ( } n=249)\end{array}$ & 12 weeks & $\begin{array}{l}\text { Patients with COPD } \\
\text { (post-bronchodilator } \\
F E V_{1}<80 \% \text { pred and } \\
F E V_{1} / F V C \text { ratio } \leqslant 0.70 \text { ) } \\
\text { Aged } \geqslant 50 \text { years } \\
\text { Smoking history of } \geqslant 10 \\
\text { pack-years }\end{array}$ & $\begin{array}{l}\text { Salmeterol/ } \\
\text { fluticasone } \\
\text { propionate } \\
\text { combination } \\
50 \mu \mathrm{g} / 250 \mu \mathrm{g} \\
\text { twice daily } \\
\text { ( } \mathrm{n}=123 \text { ) } \\
\text { Placebo (n=126) } \\
\text { (Both arms } \\
\text { received open } \\
\text { label tiotropium } \\
18 \mu \mathrm{g} \text { once daily } \\
\text { for } 4 \text { weeks after } \\
\text { a } 12 \text { week } \\
\text { treatment period) }\end{array}$ & $\begin{array}{l}\text { aPWV change from } \\
\text { baseline at } \\
12 \text { weeks }\end{array}$ & $\begin{array}{l}\text { For patients that remained } \\
\text { on treatment for } \\
12 \text { weeks ( } \mathrm{n}=96 \text { in each } \\
\text { group), salmeterol/ } \\
\text { fluticasone propionate } \\
\text { combination was } \\
\text { associated with a } \\
\text { significant reduction in } \\
\text { aPWV versus placebo } \\
\left(-0.49 \mathrm{~m} \cdot \mathrm{s}^{-1}, \mathrm{p}=0.045\right)\end{array}$ & $\begin{array}{l}\text { No significant changes in } \\
\text { aPWV for salmeterol/ } \\
\text { fluticasone propionate } \\
\text { combination }+ \\
\text { tiotropium versus } \\
\text { tiotropium from } 12- \\
16 \text { weeks }(\mathrm{mean} \\
\text { change } 0.18 \mathrm{~m} \cdot \mathrm{s}^{-1} \text { ) }\end{array}$ \\
\hline
\end{tabular}




\begin{tabular}{|c|c|c|c|c|c|c|c|}
\hline Author/study & Design and participants & Duration & Population & Treatment(s) & Primary outcome & Key results & Other findings \\
\hline $\begin{array}{l}\text { STONE } \\
\text { (2016) [91] }\end{array}$ & $\begin{array}{l}\text { Single-centre, } \\
\text { randomised, double- } \\
\text { blind, placebo- } \\
\text { controlled, crossover } \\
\text { (n=45) }\end{array}$ & $\begin{array}{l}\text { Two } 7 \text {-day } \\
\text { treatment } \\
\text { periods } \\
\text { separated } \\
\text { by a } \\
7 \pm 2 \text {-day } \\
\text { washout } \\
\text { period }\end{array}$ & $\begin{array}{l}\text { COPD (FEV } 1<70 \% \text { pred }) \\
\text { Smoking history } \\
\geqslant 15 \text { pack-years } \\
\text { Aged }>40 \text { years } \\
\text { MRC score }>1 \\
\text { Lung hyperinflation (RV } \\
>120 \% \text { pred) which } \\
\text { improved } \geqslant 7.5 \% \text { after } \\
\text { salbutamol }\end{array}$ & $\begin{array}{l}\text { Fluticasone furoate/ } \\
\text { vilanterol } \\
\text { combination } \\
100 \mu \mathrm{g} / 25 \mu \mathrm{g} \text { once } \\
\text { daily } \\
\text { Placebo }\end{array}$ & $\begin{array}{l}\text { Change in RVEDVI } \\
\text { from baseline } \\
\text { versus placebo after } \\
7 \text { days treatment } \\
\text { (maximum } 14 \text { days) }\end{array}$ & $\begin{array}{l}\text { Mean increase in change } \\
\text { from baseline in RVEDVI } \\
\text { of } 5.8 \mathrm{~mL} \cdot \mathrm{m}^{-2}(95 \% \mathrm{Cl} \\
2.74-8.91), \mathrm{p}<0.001 \\
\text { versus placebo }\end{array}$ & $\begin{array}{l}\text { Improved lung } \\
\text { hyperinflation and } \\
\text { airflow limitation from } \\
\text { baseline with } \\
\text { fluticasone furoate/ } \\
\text { vilanterol combination } \\
\text { relative to placebo, as } \\
\text { follows: } \\
\text { 1) RV: } 429 \mathrm{~mL} \\
\text { reduction (p<0.001) } \\
\text { 2) Increased IC, IC/ } \\
\text { TLC, FEV1 and FVC } \\
\text { (261 } \mathrm{mL}, 4.6 \%, 220 \mathrm{~mL} \\
\text { and } 350 \mathrm{~mL} \text {, } \\
\text { respectively; all } \\
\text { p<0.001) }\end{array}$ \\
\hline $\begin{array}{l}\text { VESTBO } \\
\text { (2016, } \\
\text { SUMMIT) } \\
\text { [92] }\end{array}$ & $\begin{array}{l}\text { Multicentre, randomised, } \\
\text { double-blind, placebo- } \\
\text { controlled, parallel } \\
\text { group, event-driven } \\
\text { (n=16485) }\end{array}$ & $\begin{array}{l}\text { Maximum } \\
\text { follow-up } \\
\text { was } 4 \text { years }\end{array}$ & $\begin{array}{l}\text { COPD } \\
\text { (post-bronchodilator } \\
\text { FEV } 150-70 \% \text { pred } \\
\text { and FEV } / \text { FVC } \leqslant 0.70 \text { ) } \\
\text { Smoking history } \\
\geqslant 10 \text { pack-years } \\
\text { mMRC dyspnoea scale } \\
\geqslant 2 \\
\text { History/increased risk of } \\
\text { CVD }\end{array}$ & $\begin{array}{l}\text { Fluticasone furoate/ } \\
\text { vilanterol } \\
\text { combination } \\
100 \mu \mathrm{g} / 25 \mu \mathrm{g} \text { once } \\
\text { daily (n=4121) } \\
\text { Fluticasone furoate } \\
100 \mu \mathrm{g} \text { once daily } \\
\text { (n=4135) } \\
\text { Vilanterol } 25 \mu \mathrm{g} \\
\text { once daily } \\
\text { (n=4118) } \\
\text { Placebo ( } \mathrm{n}=4111 \text { ) }\end{array}$ & All-cause mortality & $\begin{array}{l}\text { All-cause mortality did not } \\
\text { differ significantly } \\
\text { between fluticasone } \\
\text { furoate/vilanterol } \\
\text { combination and } \\
\text { placebo (HR } 0.88(95 \% \\
\text { Cl } 0.74-1.04) ; 12 \% \\
\text { relative reduction, } \\
\text { p=0.137) or components }\end{array}$ & $\begin{array}{l}\text { Fluticasone furoate/ } \\
\text { vilanterol combination } \\
\text { had no effect on } \\
\text { composite CV events } \\
\text { (CV death, MI, stroke, } \\
\text { unstable angina, TIA) } \\
\text { compared with placebo } \\
\text { (HR } 0.93,95 \% \mathrm{Cl} \\
0.75-1.14 \text { ) }\end{array}$ \\
\hline $\begin{array}{l}\text { ВНАTT } \\
\text { (2017) [93] }\end{array}$ & $\begin{array}{l}\text { Multicentre, randomised, } \\
\text { double-blind, parallel } \\
\text { group, placebo- } \\
\text { controlled (stratified by } \\
\text { COPD exacerbation } \\
\text { history) ( } \mathrm{n}=430 \text { ) }\end{array}$ & 24 weeks & $\begin{array}{l}\text { Patients aged } \geqslant 40 \text { years } \\
\text { with a history of COPD } \\
\geqslant 10 \text { pack-year smoking } \\
\text { history } \\
\text { FEV } 1 / F V C \leqslant 0.70 \\
\text { Post-bronchodilator FEV } 1 \\
\leqslant 70 \% \text { pred } \\
\text { aPWV } \geqslant 11 \mathrm{~m} \cdot \mathrm{s}^{-1}\end{array}$ & $\begin{array}{l}\text { Fluticasone furoate/ } \\
\text { vilanterol } \\
\text { combination } \\
100 \mu \mathrm{g} / 25 \mu \mathrm{g} \text { once } \\
\text { daily }(\mathrm{n}=135) \\
\text { Vilanterol } 25 \mu \mathrm{g} \\
\text { once daily }(\mathrm{n}=154) \\
\text { Placebo ( } \mathrm{n}=141)\end{array}$ & $\begin{array}{l}\text { Change from baseline } \\
\text { in aPWV after } \\
24 \text { weeks with } \\
\text { fluticasone furoate/ } \\
\text { vilanterol } \\
\text { combination versus } \\
\text { placebo }\end{array}$ & $\begin{array}{l}\text { No significant difference in } \\
\text { mean change from } \\
\text { baseline in aPWV at } \\
24 \text { weeks with } \\
\text { fluticasone furoate/ } \\
\text { vilanterol combination } \\
\left(-1.75 \mathrm{~m} \cdot \mathrm{s}^{-1}\right) \text { versus } \\
\text { placebo }\left(-1.97 \mathrm{~m} \cdot \mathrm{s}^{-1}\right)\end{array}$ & $\begin{array}{l}\text { Post-hoc analysis } \\
\text { indicated a greater } \\
\text { proportion of } \\
\text { responders }^{f} \text { in the } \\
\text { fluticasone furoate/ } \\
\text { vilanterol combination } \\
\text { versus placebo groups } \\
\text { when withdrawn } \\
\text { patients were } \\
\text { classified as } \\
\text { non-responders }(50 \% \\
\text { versus 36\%, } \\
\text { respectively) }\end{array}$ \\
\hline
\end{tabular}


TABLE 1 Continued

\section{Author/study Design and participants Duration}

Population

Treatment(s)

Primary outcome

Key results

Other findings

\section{Other COPD}

pharmacological

treatments

SuISSA

Population-based cohort

(1996) [94] from Saskatchewan

Canada (n=12301) Patients with asthma
aged $5-54$ years

Theophylline and $\beta$-agonists versus control

Identified $30 \mathrm{CV}$

deaths in which

acute asthma did

not appear to be a contributing factor

cohort, UK General

Database after January

$01,1994)(n=5710)$

WHITE

Pooled analysis of 14

Range: 12-

intermediate and

52 weeks

long-term trials $(\mathrm{n}=12$

054)
Patients aged $10-79$ years Theophylline land with asthma or COPD other therapies: (710 cases and $5000 \quad \beta$-agonists, oral controls]

Rhythm disorders

$$
\text { steroids, ICS) }
$$

Patients with moderateto-very-severe COPD

\section{Roflumilast}

( $n=6563$ )

Placebo $(n=5491)$
MACE ICV death,
non-fatal $\mathrm{MI}$ and
strokel

Rate of CV death was

greater with

theophylline (rate ratio

2.7. 95\% Cl 1.2-6.1) and

$\beta$-agonists administered

orally or by nebuliser

(rate ratio $2.4,95 \% \mathrm{Cl}$

1.0-5.4)

Short-term theophylline

use was weakly

associated with:

1) Arrhythmia (relative

risk $1.8,95 \% \mathrm{Cl} 1.0-3.3)$

2) $\mathrm{AF}$ (relative risk 1.8,

95\% Cl 0.9-3.7)

MACE composite rate was significantly lower with roflumilast versus

placebo (HR $0.65195 \%$

Cl 0.45-0.93), $p=0.019 \mid$
Rate of CV death was not greater with $\beta$-agonists administered by MDI

(rate ratio $1.2,95 \% \mathrm{Cl}$ 0.5-2.7)

Short-term theophylline use was associated with supraventricular

tachycardia Irelative

risk $4.0,95 \% \mathrm{C}$

0.9-18.1)

MACE experienced by $14.3 / 1000$

patient-years

(roflumilast) and by

22.3/1000

patient-years (placebo)

ACS: acute coronary syndrome; AE: adverse event; AF: atrial fibrillation; aPWV: aortic pulse wave velocity; BP: blood pressure; CAD: coronary artery disease; CCV: cardiovascular and cerebrovascular; CPRD: Clinical Practice Research Datalink; CVD: cardiovascular disease; DT-TR: tricuspid E-wave deceleration time; ECOG: Eastern Cooperative Oncology Group; ED: emergency department; E/e': ratio of peak early diastolic mitral flow velocity to peak early diastolic mitral annual movement velocity; FEV1: forced expiratory volume in 1 s; FRC: functional residual capacity; FVC: forced vital capacity; HES: hospital episode statistics; HF: heart failure; HR: hazard ratio; IC: inspiratory capacity; ICS: inhaled corticosteroid; IRV: inspiratory reserve volume; LABA: long-acting $\beta_{2}$-agonist: LAMA: long-acting muscarinic antagonist; LVEDV: left-ventricular end-diastolic volume; LVEF: left-ventricular ejection fraction; MACE: major adverse cardiovascular event; MDI: metered dose inhaler; MI: myocardial infarction; mMRC: modified Medical Research Council; MRI: magnetic resonance imaging; NS: not significant; OR: odds ratio; $\mathrm{PaO}_{2}$ : arterial oxygen tension; PASP: pulmonary arterial systolic pressure; QT: cardiac output; RCT: randomised controlled trial; RVEDVI: right-ventricular end-diastolic volume index; RV: residual volume; SABA: short-acting $\beta_{2}$-agonist; SAE: serious adverse event; SFC: salmeterol/fluticasone combination; SBP: systolic blood pressure; SPAP: systolic pulmonary arterial pressure; SV: stroke volume; TAPSE: tricuspid annular plane systolic excursion; TIA: transient ischaemic attack; TLC: total lung capacity; VC: vital

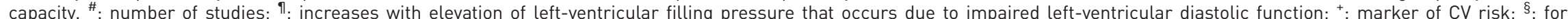
each case, one control was randomly selected, matched for age ( \pm 1 year), sex, duration of COPD, HF and history of hospitalisation for ACS, HF, ischaemic stroke, cardiac arrhythmia and acute respiratory disease lacute exacerbation of COPD, pneumonia, influenza, or acute bronchitis); $f^{f}$ patients with aPWV reduction from baseline of $\geqslant 1 \mathrm{~m} \cdot \mathrm{s}^{-1}$ on day 168 . 


\begin{tabular}{|c|c|c|c|c|c|c|c|}
\hline Author/study & $\begin{array}{l}\text { Design and } \\
\text { participants }\end{array}$ & Duration & Population & Treatment(s) & Primary outcome & Key results & Other findings \\
\hline \multicolumn{8}{|l|}{ B-blockers } \\
\hline $\begin{array}{l}\text { DRANSFIELD } \\
\text { (2008) [97] }\end{array}$ & $\begin{array}{l}\text { Retrospective, cohort } \\
\text { (University of } \\
\text { Alabama Hospital) } \\
\text { (n=825) }\end{array}$ & & $\begin{array}{l}\text { Discharge or death summaries } \\
\text { indicated primary diagnosis of } \\
\text { AECOPD or primary diagnosis } \\
\text { of ARF and secondary } \\
\text { diagnosis of AECOPD }\end{array}$ & $\begin{array}{l}\text { Users of } \beta \text {-blockers } \\
\quad(n=142) \\
\text { Non-users }(n=683)\end{array}$ & $\begin{array}{l}\text { In-hospital } \\
\text { mortality }\end{array}$ & $\begin{array}{l}\beta \text {-blocker use was } \\
\text { associated with a } \\
\text { reduction in mortality } \\
\text { (OR } 0.39,95 \% \mathrm{Cl} \\
0.14-0.99 \text { ) } \\
\text { A significant association } \\
\text { was observed between } \\
\text { daily } \beta \text {-blocker doses } \\
\text { and mortality (OR 0.31, } \\
95 \% \mathrm{Cl} 0.12-0.80 \text { ) }\end{array}$ & $\begin{array}{l}\text { SABA use was also } \\
\text { associated with a } \\
\text { reduction in } \\
\text { mortality (OR 0.39, } \\
95 \% \mathrm{Cl} 0.14-0.99 \text { ) }\end{array}$ \\
\hline $\begin{array}{l}\text { RUTTEN (2010; } \\
\text { Utrecht GP } \\
\text { Network } \\
\text { Database) [98] }\end{array}$ & $\begin{array}{l}\text { Observational, cohort } \\
\qquad(\mathrm{n}=2230)\end{array}$ & & $\begin{array}{l}\text { Individuals aged } \geqslant 45 \text { years with } \\
\text { an incident or prevalent } \\
\text { diagnosis of COPD }\end{array}$ & $\beta$-blockers & $\begin{array}{l}\text { All-cause mortality } \\
\text { First COPD } \\
\quad \text { exacerbation }\end{array}$ & $\begin{array}{l}\beta \text {-blocker use was } \\
\text { associated with: } \\
\text { 1) A reduction in } \\
\text { mortality (HR 0.68, } \\
95 \% \mathrm{Cl} 0.56-0.83 \text { ) } \\
\text { 2) A reduction in } \\
\text { exacerbations (HR } 0.71 \text {, } \\
95 \% \mathrm{Cl} 0.60-0.83 \text { ) }\end{array}$ & $\begin{array}{l}\text { Subgroup analyses } \\
\text { revealed that } \\
\text { patients with COPD } \\
\text { but without overt } \\
\text { CVD had similar } \\
\text { results }\end{array}$ \\
\hline $\begin{array}{l}\text { SHORT (2011) } \\
\text { [99] }\end{array}$ & $\begin{array}{l}\text { Retrospective, cohort } \\
\text { (NHS Tayside } \\
\text { Respiratory } \\
\text { Disease } \\
\text { Information } \\
\text { System) (n=5977) }\end{array}$ & & $\begin{array}{l}\text { Diagnosis of COPD (GOLD } \\
\text { guidelines) }\end{array}$ & $\begin{array}{l}\text { Respiratory and CV } \\
\text { drugs }\end{array}$ & $\begin{array}{l}\text { Mortality } \\
\text { COPD-related } \\
\text { hospital } \\
\text { admissions }\end{array}$ & $\begin{array}{l}\beta \text {-blocker use was } \\
\text { associated with: } \\
\text { 1) A } 22 \% \text { reduction in } \\
\text { all-cause mortality } \\
\text { versus no } \beta \text {-blocker use } \\
\text { 2) Reduction in mortality } \\
\text { from } \mathrm{Ml}(\mathrm{HR} 0.67,95 \% \mathrm{Cl} \\
0.41-1.10 \text { ) and COPD (HR } \\
0.88,95 \% \mathrm{Cl} 0.32-2.38) \\
\text { 3) Reduced risk of } \\
\text { respiratory-related } \\
\text { hospital admissions (HR } \\
0.31,95 \% \mathrm{Cl} 0.22-0.44)\end{array}$ & \\
\hline $\begin{array}{l}\text { Stefan (2012) } \\
{[100]}\end{array}$ & $\begin{array}{l}\text { Retrospective, cohort } \\
\text { (Perspective } \\
\text { Inpatient } \\
\text { Administrative } \\
\text { Database (Premier } \\
\text { Inc, Charlotte, NC, } \\
\text { USA), } 404 \\
\text { hospitals) (n=35 } \\
\text { 082) }\end{array}$ & & $\begin{array}{l}\text { Individuals aged } \geqslant 40 \text { years with } \\
\text { a principal diagnosis of } \\
\text { AECOPD, or a principal } \\
\text { diagnosis of respiratory failure } \\
\text { and a secondary diagnosis of } \\
\text { AECOPD or emphysema, and } \\
\text { with a secondary diagnosis of } \\
\text { IHD or HF }\end{array}$ & $\begin{array}{l}\beta \text {-blockers } \\
\text { Treatment with } \\
\text { inhaled } \\
\beta_{2} \text {-agonists and } \\
\text { systemic } \\
\text { corticosteroids on } \\
\text { the first or second } \\
\text { day of the } \\
\text { hospitalisation }\end{array}$ & $\begin{array}{l}\text { In-hospital } \\
\text { mortality }\end{array}$ & $\begin{array}{l}\text { No association between } \\
\beta \text {-blocker therapy and: } \\
\text { 1) In-hospital mortality } \\
\text { (OR 0.88, } 95 \% \mathrm{Cl} \\
0.71-1.09 \text { ) } \\
\text { 2) } 30 \text {-day readmission } \\
\text { (OR } 0.96,95 \% \mathrm{Cl} \\
\text { 0.89-1.03) } \\
\text { 3) Late mechanical } \\
\text { ventilation (OR 0.98, } \\
95 \% \mathrm{Cl} 0.77-1.24 \text { ) }\end{array}$ & $\begin{array}{l}25 \% \text { increased odds of } \\
30 \text {-day readmission } \\
\text { (OR } 1.25,95 \% \mathrm{Cl} \\
1.08-1.44 \text { ) with } \\
\text { nonselective } \\
\beta \text {-blocker versus } \\
\beta_{1} \text {-selective } \\
\beta \text {-blocker }\end{array}$ \\
\hline
\end{tabular}




\begin{tabular}{|c|c|c|c|c|c|c|}
\hline Author/study & $\begin{array}{l}\text { Design and } \\
\text { participants }\end{array}$ & Duration & Population & Treatment(s) & Primary outcome & Other findings \\
\hline $\begin{array}{l}\text { Du (2014) } \\
{[101]}\end{array}$ & $\begin{array}{l}\text { Meta-analysis (15 } \\
\text { observational, } \\
\text { cohort studies) } \\
\text { ( } n=121956 \text { ) }\end{array}$ & $1-7.2$ years & Individuals with COPD & $\beta$-blockers & Mortality & $\begin{array}{l}\beta \text {-blocker use was } \\
\text { associated with: } \\
\text { 1) A reduction in COPD } \\
\text { exacerbations (rate ratio } \\
0.63,95 \% \mathrm{Cl} 0.57-0.71 \text { ) } \\
\text { 2) A reduction in the risk } \\
\text { of overall mortality (rate } \\
\text { ratio } 0.72,95 \% \mathrm{Cl} \\
0.63-0.83 \text { ) }\end{array}$ \\
\hline $\begin{array}{l}\text { Puente-Maestu } \\
\text { (2014) [102] }\end{array}$ & $\begin{array}{l}\text { Analytical, } \\
\text { cross-sectional } \\
(n=256)\end{array}$ & & $\begin{array}{l}\text { Individuals with previous COPD } \\
\text { diagnosis and CHF/CAD } \\
\text { diagnosis } \geqslant 1 \text { year prior to } \\
\text { baseline who meet the criteria } \\
\text { for } \beta \text {-blocker treatment with } \\
\text { no contraindications }\end{array}$ & $\beta$-blocker & $\begin{array}{l}\text { Lung function } \\
\text { ECG } \\
\text { LVEF } \\
\text { Haemoglobin } \\
\text { concentrations } \\
\text { Heart rate } \\
\text { Exacerbations } \\
\text { Hospital } \\
\text { admissions } \\
\text { CAT } \\
\text { Comorbidities }\end{array}$ & $\begin{array}{l}\beta \text {-blocker use was } \\
\text { associated with: } \\
\text { 1) Fewer COPD patients } \\
\text { experiencing } \\
\text { exacerbations requiring } \\
\text { ER visits ( } 36.9 \% \text { versus } \\
58.8 \% \text { among patients } \\
\text { without } \beta \text {-blockers, } \\
\text { p<0.000) } \\
\text { 2) Fewer COPD patients } \\
\text { experiencing } \geqslant 2 \\
\text { exacerbations or ER visits } \\
\text { (38.8\% versus } 58.8 \% \\
\text { among patients without } \\
\beta \text {-blockers, } p<0.000)\end{array}$ \\
\hline $\begin{array}{l}\text { B HATT (2016; } \\
\text { follow-up of } \\
\text { the COPDGene } \\
\text { cohort) [103] }\end{array}$ & $\begin{array}{l}\text { Prospective, } \\
\text { follow-up (n=3464) }\end{array}$ & $\begin{array}{l}2.1 \text { years } \\
\text { median } \\
\text { follow-up }\end{array}$ & $\begin{array}{l}\text { Individuals diagnosed with GOLD } \\
\text { stage } 2 \text { to } 4 \text { COPD }\end{array}$ & $\begin{array}{l}\beta \text {-blocker } \\
\text { CCB } \\
\text { ACEI/ARB }\end{array}$ & $\begin{array}{l}\text { Exacerbation rate } \\
\quad \text { (total and severe) }\end{array}$ & $\begin{array}{l}\beta \text {-blocker use was associated } \\
\text { with a reduction in the rate } \\
\text { of total exacerbations } \\
\text { (IRR } 0.73(95 \% \mathrm{Cl} 0.60 \text { - } \\
0.90), \mathrm{p}=0.003 \text { ) and severe } \\
\text { exacerbations (IRR } 0.67 \\
(95 \% \mathrm{Cl} 0.48-0.93), \\
\mathrm{p}=0.016 \text { ) } \\
\text { In individuals with GOLD } \\
\text { stage } 3 \text { and } 4, \beta \text {-blocker } \\
\text { use was associated with a } \\
\text { reduction in the rate of } \\
\text { total exacerbations (IRR } \\
0.33 \text { (95\% Cl } 0.19-0.58 \text { ), } \\
\mathrm{p}<0.001 \text { ) and severe } \\
\text { exacerbations (IRR } 0.35 \\
\text { (95\% CI } 0.16-0.76 \text { ), } \\
\mathrm{p}=0.008 \text { ) }\end{array}$ \\
\hline $\begin{array}{l}\text { KEY (2017) } \\
{[104]}\end{array}$ & $\begin{array}{l}\text { Cohort, cross-over } \\
\qquad(\mathrm{n}=48)\end{array}$ & & $\begin{array}{l}\text { Individuals were aged } \geqslant 18 \text { years } \\
\text { and able to perform a } \\
\text { cardiopulmonary exercise test }\end{array}$ & $\beta$-blocker & Lung function & $\begin{array}{l}\beta \text {-blocker use led to a } \\
\text { small reduction in FEV } 1 \\
\text { compared with non-use }\end{array}$ \\
\hline
\end{tabular}




\begin{tabular}{|c|c|c|c|c|c|c|c|}
\hline Author/study & $\begin{array}{l}\text { Design and } \\
\text { participants }\end{array}$ & Duration & Population & Treatment(s) & Primary outcome & Key results & Other findings \\
\hline \multicolumn{8}{|l|}{$\begin{array}{l}\text { RAAS blockers } \\
\text { (ACEIs, ARBs) }\end{array}$} \\
\hline $\begin{array}{l}\text { KaNAZAWA } \\
\text { (2003) [105] }\end{array}$ & $\begin{array}{l}\text { Randomised, double- } \\
\text { blind, placebo- } \\
\text { controlled, } \\
\text { crossover, pilot } \\
\text { (n=36) }\end{array}$ & & $\begin{array}{l}\text { Males with COPD (ACE } \\
\text { genotypes II (n=13), ID (n=11), } \\
\text { DD (n=12)) }\end{array}$ & $\begin{array}{l}\text { Captopril } \\
25 \mathrm{mg} \cdot \text { day }^{-1} \\
\text { Placebo }\end{array}$ & $\begin{array}{l}\text { Pulmonary } \\
\text { haemodynamics } \\
\text { (mean PAP, PVR, } \\
\text { lactate } \\
\text { concentration } \\
\text { and } \mathrm{PvO}_{2} \text { ) }\end{array}$ & $\begin{array}{l}\text { Mean PAP, PVR and lactate } \\
\text { concentration after } \\
\text { exercise were lower for } \\
\text { captopril than placebo in } \\
\text { patients with the } \\
\text { genotypes II or ID }\end{array}$ & $\begin{array}{l}\mathrm{PvO}_{2} \text { after exercise was } \\
\text { higher with } \\
\text { captopril versus } \\
\text { placebo in patients } \\
\text { with genotype II }\end{array}$ \\
\hline $\begin{array}{l}\text { ANDREAS (2006) } \\
{[106]}\end{array}$ & $\begin{array}{l}\text { Randomised, double- } \\
\text { blind, placebo- } \\
\text { controlled }(n=60)\end{array}$ & 4 months & $\begin{array}{l}\text { Patients with COPD (FEV1 } \\
<50 \% \text { pred) } \\
\text { Aged } 30-80 \text { years }\end{array}$ & $\begin{array}{l}\text { Irbesartan } \\
150 \mathrm{mg}^{- \text {day }^{-1}} \\
\text { (increased to } \\
300 \mathrm{mg} \cdot \text { day }^{-1} \text { after } \\
4 \text { weeks) }(\mathrm{n}=30) \\
\text { Placebo }(\mathrm{n}=30)\end{array}$ & $\begin{array}{l}\text { Lung function } \\
\text { (PImax })\end{array}$ & $\begin{array}{l}\text { Irbesartan did not affect } \\
\text { PImax (baseline: } 4.8 \mathrm{kPa} \\
4 \text { months: } 4.5 \mathrm{kPa} \text { ) }\end{array}$ & $\begin{array}{l}\text { Irbesartan reduced: } \\
\text { 1) TLC (baseline: } \\
119.7 \% \text { pred; } \\
4 \text { months: } 113.7 \% \\
\text { pred; } p=0.01 \text { ) } \\
\text { 2) Haematocrit } \\
\text { (from } 46.4 \% \text { to } \\
43.9 \%, p<0.0001 \\
\text { versus placebo) }\end{array}$ \\
\hline $\begin{array}{l}\text { PARIKH (2017) } \\
\text { [124] }\end{array}$ & $\begin{array}{l}\text { Population-based } \\
\text { cohort study las } \\
\text { part of the } \\
\text { Multi-Ethnic Study } \\
\text { of Atherosclerosis) } \\
\text { (n=4472) }\end{array}$ & $\begin{array}{l}9.3 \text { years } \\
\text { median } \\
\text { follow-up }\end{array}$ & $\begin{array}{l}\text { Participants aged } 45-84 \text { years } \\
\text { from the general population } \\
\text { (3\% had emphysema at } \\
\text { baseline) }\end{array}$ & $\begin{array}{l}\text { ACEls } \\
\text { ARBs }\end{array}$ & $\begin{array}{l}\text { Percent } \\
\text { emphysema } \\
\text { (percentage of } \\
\text { lung regions less } \\
\text { than }-950 \\
\text { Hounsfield units } \\
\text { on CT scans) }\end{array}$ & $\begin{array}{l}\text { Higher doses of ACE or } \\
\text { ARB were independently } \\
\text { associated with a slower } \\
\text { change in percent } \\
\text { emphysema ( } p=0.03 \text { ). } \\
\text { Over } 10 \text { years, the } \\
\text { predicted mean increase } \\
\text { in participants who used } \\
\text { maximum doses of ARBs } \\
\text { or ACEls was } 0.06 \\
\text { percentage points versus } \\
\text { 0.66 percentage points in } \\
\text { those who did not take } \\
\text { ARBs or ACEls ( } p=0.01 \text { ) }\end{array}$ & $\begin{array}{l}\text { The findings were of } \\
\text { greatest magnitude } \\
\text { among former } \\
\text { smokers }(p<0.001)\end{array}$ \\
\hline $\begin{array}{l}\text { LAI (2018) } \\
{[122]}\end{array}$ & $\begin{array}{l}\text { Population-based } \\
\text { cohort (Taiwan } \\
\text { National Health } \\
\text { Insurance } \\
\text { Database) ( } n=12 \\
\text { 452) }\end{array}$ & $\begin{array}{l}\text { 6-11 year } \\
\text { follow-up }\end{array}$ & $\begin{array}{l}\text { Patients with COPD aged } \\
\geqslant 40 \text { years who received } \\
\text { prescriptions for an ACEI or } \\
\text { ARB for }>90 \text { days between } \\
2000 \text { and } 2005 \\
\text { Allocated to ACEI ( } n=6226 \text { ) and } \\
\text { ARB ( } n=6226 \text { ) cohorts }\end{array}$ & $\begin{array}{l}\text { ACEls } \\
\text { ARBs }\end{array}$ & $\begin{array}{l}\text { Pneumonia } \\
\text { Severe } \\
\text { exacerbations } \\
\text { (COPD-related } \\
\text { hospitalisation or } \\
\text { ER visit) } \\
\text { Mortality }\end{array}$ & $\begin{array}{l}\text { Patients treated with ACEls } \\
\text { had significantly higher } \\
\text { rates of severe COPD } \\
\text { exacerbations ladjusted } \\
\text { rate ratio } 1.22,95 \% \mathrm{Cl} \\
1.15-1.29 \text { ) and a higher } \\
\text { risk of pneumonia } \\
\text { (adjusted HR } 1.22,95 \% \\
\mathrm{Cl} 1.15-1.29 \text { ) than those } \\
\text { in the ARB group }\end{array}$ & $\begin{array}{l}\text { ARBs were also } \\
\text { associated with a } \\
\text { lower risk of } \\
\text { pneumonia } \\
\text { requiring } \\
\text { mechanical } \\
\text { ventilation ladjusted } \\
\text { HR } 1.35,95 \% \mathrm{Cl} \\
\text { 1.24-1.47) and of } \\
\text { mortality ladjusted } \\
\text { HR } 1.33,95 \% \mathrm{Cl} \\
1.26-1.42 \text { l }\end{array}$ \\
\hline
\end{tabular}




\begin{tabular}{|c|c|c|c|c|c|c|c|}
\hline Author/study & $\begin{array}{l}\text { Design and } \\
\text { participants }\end{array}$ & Duration & Population & Treatment(s) & Primary outcome & Key results & Other findings \\
\hline \multicolumn{8}{|l|}{ Statins } \\
\hline $\begin{array}{l}\text { MANCINI (2006) } \\
{[107]}\end{array}$ & $\begin{array}{l}\text { Retrospective, } \\
\text { time-matched } \\
\text { nested case- } \\
\text { control (Quebec } \\
\text { Linked Databases) } \\
\text { ( } \mathrm{n}=19720 \text { ) }\end{array}$ & & $\begin{array}{l}\text { Two cohorts (aged } \geqslant 65 \text { years) as } \\
\text { follows: } \\
\text { 1) Revascularised patients } \\
\text { with high CV risk ( }=946 \\
\text { cases; } n=18774 \text { controls) } \\
\text { 2) General population of } \\
\text { NSAID users without previous } \\
\text { MI ( } n=4907 \text { cases; } n=98097 \\
\text { controls) }\end{array}$ & $\begin{array}{l}\text { Statins } \\
\text { ACEls } \\
\text { ARBs }\end{array}$ & $\begin{array}{l}\text { COPD } \\
\text { hospitalisation } \\
\text { MI } \\
\text { Mortality }\end{array}$ & $\begin{array}{l}\text { Statin use was associated } \\
\text { with reduced risk for } \\
\text { COPD hospitalisation } \\
\text { ( } p=0.0091 \text { ) and with the } \\
\text { combined use of statins } \\
\text { and ACEls or ARBs } \\
\text { ( } p=0.0012 \text { ) } \\
\text { Risk ratios for MI were } \\
\text { reduced by all three drug } \\
\text { classes, particularly by } \\
\text { the combination of } \\
\text { statins with ACEls or } \\
\text { ARBs ( } p<0.0001) \\
\text { Death risk ratios were } \\
\text { reduced by ARBs } \\
\text { ( } p=0.0010) \text {, statins } \\
(p<0.0001) \text { and statins } \\
\text { with ACEls or ARBs } \\
(p<0.0001)\end{array}$ & $\begin{array}{l}\text { Similar benefits were } \\
\text { observed when } \\
\text { steroid users were } \\
\text { included in the } \\
\text { analysis }\end{array}$ \\
\hline $\begin{array}{l}\text { ALEXEEFF (2007; } \\
\text { Veterans } \\
\text { Administration } \\
\text { normative } \\
\text { study) [108] }\end{array}$ & Longitudinal (n=803) & $\begin{array}{l}10 \text { year } \\
\text { follow-up }\end{array}$ & $\begin{array}{l}\text { Elderly men with no prior known } \\
\text { medical conditions }\end{array}$ & Statins & $\begin{array}{r}\text { Lung function } \\
\text { (FEV1, FVC) }\end{array}$ & 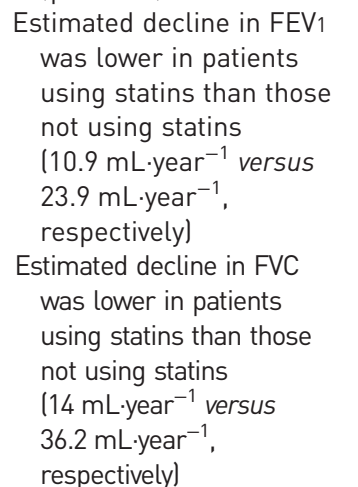 & $\begin{array}{l}\text { There was a } \\
\text { significant } \\
\text { three-way } \\
\text { association between } \\
\text { time since first visit, } \\
\text { statin use and } \\
\text { smoking status } \\
(p<0.001)\end{array}$ \\
\hline $\begin{array}{l}\text { LeE (2009) } \\
\text { [109] }\end{array}$ & $\begin{array}{l}\text { Randomised, double- } \\
\text { blind, parallel } \\
\text { group }(n=53)\end{array}$ & 6 months & $\begin{array}{l}\text { Patients with COPD and } \mathrm{PH} \text { aged } \\
\quad 40-80 \text { years } \\
\text { FEV } 1<80 \% \text { pred } \\
\mathrm{FEV}_{1} / \mathrm{FVC}<70 \%\end{array}$ & $\begin{array}{l}\text { Pravastatin } \\
\quad \begin{array}{l}40 \mathrm{mg} \cdot \text { day }^{-1} \\
(\mathrm{n}=27)\end{array} \\
\text { Placebo }(\mathrm{n}=26)\end{array}$ & $\begin{array}{l}\text { Change in exercise } \\
\text { time from } \\
\text { baseline to } \\
6 \text { months }\end{array}$ & $\begin{array}{l}\text { Exercise time significantly } \\
\text { increased from baseline } \\
\text { with pravastatin }(52 \% \text { at } \\
6 \text { months, from } 660 \mathrm{~s} \text { to } \\
1006 \mathrm{~s} ; \mathrm{p}<0.0001)\end{array}$ & $\begin{array}{l}\text { Pravastatin was } \\
\text { associated with less } \\
\text { dyspnoea after } \\
\text { exercise versus } \\
\text { placebo (Borg } \\
\text { dyspnoea score } \\
\text { decreased from } 6.7 \\
\text { at baseline to } 3.86 \\
\text { at } 6 \text { months with } \\
\text { pravastatin versus } \\
6.9 \text { to } 6.8 \text { with } \\
\text { placebo; } p<0.05 \text { ) }\end{array}$ \\
\hline
\end{tabular}




\begin{tabular}{|c|c|c|c|c|c|c|c|}
\hline Author/study & $\begin{array}{l}\text { Design and } \\
\text { participants }\end{array}$ & Duration & Population & Treatment(s) & Primary outcome & Key results & Other findings \\
\hline $\begin{array}{l}\text { MoRTENSEN } \\
\text { (2009) [110] }\end{array}$ & $\begin{array}{l}\text { Retrospective } \\
\text { national cohort (VA } \\
\text { administrative data) } \\
\text { (n=11212) }\end{array}$ & & $\begin{array}{l}\text { Patients ( } 98 \% \text { male) aged } \\
>65 \text { years hospitalised with } \\
\text { COPD exacerbation and } \\
\text { received one or more } \\
\text { respiratory medications } \\
\text { within } 90 \text { days of presentation }\end{array}$ & $\begin{array}{l}\text { Users of statins or } \\
\text { ACEIs/ARBs } \\
\text { (n=4711) } \\
\text { Non-users ( } n=6501)\end{array}$ & 90-day mortality & $\begin{array}{l}\text { Statin use associated with } \\
\text { significantly reduced } \\
90 \text {-day mortality (OR } \\
0.51,95 \% \mathrm{Cl} 0.40-0.64 \text { ) }\end{array}$ & $\begin{array}{l}\text { ACEI/ARB use } \\
\text { associated with } \\
\text { significantly } \\
\text { reduced } 90 \text {-day } \\
\text { mortality (OR 0.55, } \\
95 \% \mathrm{Cl} 0.46-0.66 \text { ) }\end{array}$ \\
\hline $\begin{array}{l}\text { BARTZIOKAS } \\
\text { (2011) [111] }\end{array}$ & $\begin{array}{l}\text { Prospective follow-up } \\
\quad(n=245)\end{array}$ & 12 months & $\begin{array}{l}\text { Patients with COPD admitted to } \\
\text { hospital for COPD } \\
\text { exacerbations }\end{array}$ & Statins & $\begin{array}{l}\text { 30-day or 1-year } \\
\text { mortality }\end{array}$ & $\begin{array}{l}\text { Statins had no effect on } \\
\text { 30-day or 1-year } \\
\text { mortality }\end{array}$ & $\begin{array}{l}\text { Statins were } \\
\text { associated with a } \\
\text { lower risk for COPD } \\
\text { exacerbations (HR } \\
0.656,95 \% \mathrm{Cl} \\
0.454-0.946 \text { ) and } \\
\text { severe exacerbations } \\
\text { (HR } 0.608,95 \% \mathrm{Cl} \\
0.381-0.972 \text { ) }\end{array}$ \\
\hline $\begin{array}{l}\text { HuANg (2011) } \\
\text { [112] }\end{array}$ & $\begin{array}{l}\text { Population-based } \\
\text { cohort (Taiwan } \\
\text { National Health } \\
\text { Insurance } \\
\text { Database) ( } \mathrm{n}=18 \\
\text { 721) }\end{array}$ & $\begin{array}{l}4.58 \text { year mean } \\
\text { follow-up }\end{array}$ & $\begin{array}{l}\text { Patients newly diagnosed with } \\
\text { COPD (median age } 64 \text { years) } \\
\text { receiving statins for } \\
\text { hyperlipidaemia }\end{array}$ & $\begin{array}{l}\text { Statins ( } n=6252 \text { ) } \\
\text { Control ( } n=12469 ; \\
\text { matched for age, } \\
\text { sex and COPD } \\
\text { treatment } \\
\text { (non-statin users)) }\end{array}$ & $\begin{array}{l}\text { Hospitalisation for } \\
\text { COPD }\end{array}$ & $\begin{array}{l}\text { Fewer patients in the statin } \\
\text { group ( } n=508,8.1 \% \text { ) were } \\
\text { hospitalised for COPD } \\
\text { exacerbation versus the } \\
\text { control group ( } n=1324 \text {, } \\
10.6 \% \text {; } p<0.001 \text { ) }\end{array}$ & $\begin{array}{l}\text { Statin use was } \\
\text { associated with } \\
\text { decreased risk of } \\
\text { COPD } \\
\text { hospitalisation (HR } \\
0.66(95 \% \mathrm{Cl} 0.60- \\
0.74), \mathrm{p}<0.001)\end{array}$ \\
\hline $\begin{array}{l}\text { BANDo (2012; } \\
\text { Japan) [113] }\end{array}$ & $\begin{array}{l}\text { Observational, } \\
\text { cross-sectional } \\
(n=853)\end{array}$ & & $\begin{array}{l}\text { Outpatients } \geqslant 40 \text { years who } \\
\text { regularly visited a primary } \\
\text { healthcare facility }\end{array}$ & Statins & Lung function & $\begin{array}{l}\text { The prevalence of airflow } \\
\text { limitation was lower } \\
\text { among patients with a } \\
\text { history of statin use than } \\
\text { those who had not used } \\
\text { statins ( } 2.3 \% \text { versus } \\
10.5 \% \text {, respectively) } \\
\text { Statin use was not } \\
\text { significantly associated } \\
\text { with a lower prevalence } \\
\text { of airflow limitation }\end{array}$ & $\begin{array}{l}\text { Airflow limitation was } \\
\text { not observed in } \\
\text { patients with a } \\
\text { history of smoking } \\
\text { who had used } \\
\text { statins }\end{array}$ \\
\hline $\begin{array}{l}\text { WANG (2013) } \\
\text { [114] }\end{array}$ & $\begin{array}{l}\text { Retrospective nested } \\
\text { case-control } \\
\text { (nationwide health } \\
\text { insurance claims } \\
\text { database, Taiwan) } \\
\text { (n=14316) }\end{array}$ & & $\begin{array}{l}\text { Patients with COPD hospitalised } \\
\text { for COPD exacerbations } \\
\text { (n=1584) matched to } 5950 \\
\text { controls }\end{array}$ & Statins & COPD exacerbation & $\begin{array}{l}\text { Current use of statins } \\
\text { associated with a } 40 \% \\
\text { decreased risk of COPD } \\
\text { exacerbation (OR 0.60, } \\
95 \% \mathrm{Cl} 0.44-0.81 \text { ) }\end{array}$ & $\begin{array}{l}\text { Statins reduced risk of } \\
\text { COPD exacerbations } \\
\text { in a dose-dependent } \\
\text { manner (medium } \\
\text { average daily dose: } \\
\text { OR } 0.60,95 \% \mathrm{Cl} \\
0.41-0.89 ; \text { high daily } \\
\text { dose: OR } 0.33,95 \% \\
\text { Cl } 0.14-0.73 \text { ) }\end{array}$ \\
\hline
\end{tabular}




\begin{tabular}{|c|c|c|c|c|c|c|c|}
\hline Author/study & $\begin{array}{l}\text { Design and } \\
\text { participants }\end{array}$ & Duration & Population & Treatment(s) & Primary outcome & Key results & Other findings \\
\hline $\begin{array}{l}\text { LAHOUSSE } \\
\text { (2013; } \\
\text { Rotterdam } \\
\text { Study) [115] }\end{array}$ & $\begin{array}{l}\text { Nested case-control } \\
\quad(n=7983)\end{array}$ & & $\begin{array}{l}363 \text { patients with COPD who died } \\
\text { during follow-up versus } 2345 \\
\text { age-/sex-matched COPD } \\
\text { controls }\end{array}$ & Statins & Mortality & $\begin{array}{l}\text { Long-term statin use } \\
\text { (>2 years) was associated } \\
\text { with a } 39 \% \text { decreased } \\
\text { risk of death }\end{array}$ & $\begin{array}{l}\text { Long-term statin use } \\
\text { was associated with } \\
78 \% \text { reduced } \\
\text { mortality with } \\
\text { hsCRP }>3 \mathrm{mg} \cdot \mathrm{L}^{-1} \\
\text { (versus } 21 \% \\
\text { reduction for hsCRP } \\
\leqslant 3 \mathrm{mg} \cdot \mathrm{L}^{-1} \text { ) }\end{array}$ \\
\hline $\begin{array}{l}\text { CRINER (2014; } \\
\text { STATCOPE) } \\
\text { [116] }\end{array}$ & $\begin{array}{l}\text { Multicentre, } \\
\text { randomised, } \\
\text { parallel group, } \\
\text { placebo-controlled } \\
\text { (n=885) }\end{array}$ & $\begin{array}{l}\text { Up to } \\
36 \text { months } \\
\text { (mean } \\
\text { follow-up } \\
\sim 21 \text { months) }\end{array}$ & $\begin{array}{l}\text { Patients } 40-80 \text { years old with } \\
\text { moderate-to-severe } \mathrm{COPD} \\
\text { (post-bronchodilator } \mathrm{FEV} 1 / \mathrm{FVC} \\
<70 \% \text { and } \mathrm{FEV} 1<80 \% \text { pred) } \\
\text { and smoking history } \\
\geqslant 10 \text { pack-years } \\
\text { COPD exacerbation in the } \\
\text { previous year }\end{array}$ & $\begin{array}{l}\text { Simvastatin } 40 \mathrm{mg} \\
\text { once daily ( } \mathrm{n}=433 \text { ) } \\
\text { Placebo ( } \mathrm{n}=452)\end{array}$ & $\begin{array}{l}\text { Exacerbation rate } \\
\text { (number of } \\
\text { exacerbations per } \\
\text { person-year) }\end{array}$ & $\begin{array}{l}\text { No significant difference in } \\
\text { mean exacerbation rate } \\
\text { between simvastatin and } \\
\text { placebo (1.36 versus } 1.39 \\
\text { exacerbations per } \\
\text { person-year, } \\
\text { respectively) }\end{array}$ & $\begin{array}{l}\text { Median days to first } \\
\text { exacerbation was } \\
\text { similar for } \\
\text { simvastatin } \\
\text { (223 days) and } \\
\text { placebo ( } 231 \text { days) }\end{array}$ \\
\hline $\begin{array}{l}\text { INGEBRIGTSEN } \\
\text { (2015; } \\
\text { Copenhagen } \\
\text { General } \\
\text { Population } \\
\text { Study) [117] }\end{array}$ & $\begin{array}{l}\text { Nested case-control } \\
\quad(n=5794)\end{array}$ & 3 year follow-up & $\begin{array}{l}\text { Individuals with COPD and CRP } \\
\text { measurement matched for } \\
\text { age, sex, smoking, COPD } \\
\text { severity and comorbidity }\end{array}$ & Statins & $\begin{array}{l}\text { COPD } \\
\text { exacerbations }\end{array}$ & $\begin{array}{l}\text { Statins associated with } \\
\text { reduced risk of COPD } \\
\text { exacerbations (crude } \\
\text { analysis: OR } 0.68 \text { (95\% } \\
\text { Cl } 0.51-0.91), p=0.01 ; \\
\text { multivariate analysis: OR } \\
0.67(95 \% \mathrm{Cl} 0.48-0.92 \text { ), } \\
\mathrm{p}=0.01 \text { ) }\end{array}$ & $\begin{array}{l}\text { In a subgroup of } \\
\text { patients with the } \\
\text { most severe COPD } \\
\text { and no CV } \\
\text { comorbidity, statins } \\
\text { did not reduce risk } \\
\text { of COPD } \\
\text { exacerbations (OR } \\
1.1,95 \% \mathrm{Cl} 0.5-2.1 \text { ) }\end{array}$ \\
\hline $\begin{array}{l}\text { RossI }(2017 ; \\
\text { GISSI-HF) } \\
{[118]}\end{array}$ & $\begin{array}{l}\text { Randomised, double- } \\
\text { blind, placebo- } \\
\text { controlled }(n=1060)\end{array}$ & & & $\begin{array}{l}\text { Rosuvastatin } 10 \mathrm{mg} \\
\quad(\mathrm{n}=538) \\
\text { Placebo }(\mathrm{n}=522)\end{array}$ & All-cause mortality & $\begin{array}{l}\text { There was no significant } \\
\text { difference in all-cause } \\
\text { mortality between } \\
\text { rosuvastatin and placebo } \\
(p=0.30)\end{array}$ & $\begin{array}{l}\text { There were no } \\
\text { significant } \\
\text { differences in CV } \\
\text { death ( } p=0.88) \text {, } \\
\text { non-CV death } \\
\text { ( } p=0.09 \text { ) and } \\
\text { all-cause } \\
\text { hospitalisation } \\
\text { ( } p=0.82 \text { ) between } \\
\text { rosuvastatin and } \\
\text { placebo }\end{array}$ \\
\hline
\end{tabular}




\begin{tabular}{|c|c|c|c|c|c|c|c|}
\hline Author/study & $\begin{array}{l}\text { Design and } \\
\text { participants }\end{array}$ & Duration & Population & Treatment(s) & Primary outcome & Key results & Other findings \\
\hline \multicolumn{8}{|c|}{ Antiplatelet therapy } \\
\hline $\begin{array}{l}\text { EKSTRÖM (2013; } \\
\text { national } \\
\text { Swevedox } \\
\text { register) [119] }\end{array}$ & $\begin{array}{l}\text { Prospective, national, } \\
\text { multicentre } \\
(n=2249)\end{array}$ & $\begin{array}{l}4 \text { years prior to } \\
\text { baseline }\end{array}$ & $\begin{array}{l}\text { Individuals aged } \geqslant 45 \text { years with } \\
\text { physician-diagnosed COPD } \\
\text { treated with LTOT }\end{array}$ & $\begin{array}{l}\text { All dispensed } \\
\text { prescriptions in } \\
\text { outpatient care in } \\
\text { Sweden after July } \\
01,2005\end{array}$ & $\begin{array}{l}\text { Comorbidity and } \\
\text { in-hospital time }\end{array}$ & $\begin{array}{l}\text { Patients treated with } \\
\text { antiplatelet drugs had } \\
\text { higher BMI and more } \\
\text { CVD, diabetes mellitus } \\
\text { and renal failure than } \\
\text { patients not on } \\
\text { antiplatelets } \\
\text { The use of antiplatelet } \\
\text { drugs was associated } \\
\text { with decreased mortality } \\
\text { (HR 0.86 (95\% Cl } \\
0.75-0.99), \mathrm{p}=0.030)\end{array}$ & \\
\hline $\begin{array}{l}\text { HARRISON } \\
(2014 ; \\
\text { EXODUS } \\
\text { cohort) }[120]\end{array}$ & $\begin{array}{l}\text { Observational, cohort, } \\
\text { multicentre } \\
\text { (n=1343) }\end{array}$ & 1 year & $\begin{array}{l}\text { Individuals }>40 \text { years old } \\
\text { with spirometry-confirmed } \\
\text { COPD admitted to hospital } \\
\text { between } 2009 \text { and } 2011 \text { with } \\
\text { AECOPD }\end{array}$ & $\begin{array}{l}\text { All COPD and CV } \\
\text { medications }\end{array}$ & $\begin{array}{l}\text { 1-year all-cause } \\
\text { mortality }\end{array}$ & $\begin{array}{l}\text { Antiplatelet therapy was } \\
\text { correlated with a } \\
\text { reduction in 1-year } \\
\text { mortality (OR } 0.63 \text { (95\% } \\
\text { Cl } 0.47-0.85), p=0.003 \text { ) }\end{array}$ & $\begin{array}{l}\text { Antiplatelet therapy } \\
\text { was not correlated } \\
\text { with a reduction in } \\
\text { hospital mortality } \\
\text { ( } p=0.124), C V \\
\text { hospitalisation } \\
\text { ( } p=0.097 \text { ) or } C V \\
\text { death ( } p=0.311 \text { ) }\end{array}$ \\
\hline \multicolumn{8}{|l|}{$\begin{array}{l}\text { Other CV } \\
\text { medications }\end{array}$} \\
\hline $\begin{array}{l}\text { HERRIN (2013) } \\
\text { [121] }\end{array}$ & $\begin{array}{l}\text { Observational, cohort } \\
\text { (n=7104) }\end{array}$ & $\begin{array}{l}\text { Receiving care } \\
\text { between } \\
\text { January } 2001 \\
\text { and December } \\
2006 \\
\text { Follow-up April } \\
2009\end{array}$ & $\begin{array}{l}\text { Individuals with COPD and } \\
\text { hypertension prescribed with } \\
\text { two antihypertensive } \\
\text { medications }\end{array}$ & $\begin{array}{c}\text { Thiazide diuretic } \\
\text { plus } \beta \text {-blocker } \\
\text { Thiazide diuretic } \\
\text { plus ACEI/ARB } \\
\text { Thiazide diuretic } \\
\text { plus CCB } \\
\beta \text {-blocker plus } \\
\text { ACEI/ARB }\end{array}$ & $\begin{array}{l}\text { CHF (time to first } \\
\text { event requiring } \\
\text { hospitalisation) }\end{array}$ & $\begin{array}{l}\text { Choice of antihypertensive } \\
\text { medications in } \\
\text { combination with a } \\
\text { thiazide diuretic had no } \\
\text { significant effect on the } \\
\text { risk of COPD } \\
\text { exacerbations }\end{array}$ & \\
\hline
\end{tabular}

ACE: angiotensin-converting enzyme; ACEI: ACE inhibitor; AECOPD: acute exacerbation of COPD; ARB: angiotensin receptor blocker; ARF: acute respiratory failure; BMI: body mass index; CAD: coronary artery disease; CAT: COPD Assessment Test; CCB: calcium channel blocker; CHF: congestive heart failure; CRP: C-reactive protein; CT: computed tomography; CVD: cardiovascular disease; ER: emergency room; FEV1: forced expiratory volume in 1 s; FVC: forced vital capacity; GOLD, Global Initiative for Chronic Obstructive Lung Disease; HF: heart failure; HR: hazard ratio; hsCRP: high-sensitivity C-reactive protein; IHD: ischaemic heart disease; IRR: incidence risk ratio; LTOT: long-term oxygen therapy; LVEF: left-ventricular ejection fraction; MI: myocardial infarction; NHS: National Health Service; NSAID: non-steroidal anti-inflammatory drug; OR: odds ratio; PAP: pulmonary arterial pressure; PImax: maximal inspiratory pressure; PH: pulmonary hypertension; PVR: pulmonary vascular resistance; $\mathrm{PvO}_{2}$ : mixed venous oxygen tension; RAAS: renin-angiotensin-aldosterone system; SABA: shortacting $\beta_{2}$-agonist; TLC: total lung capacity; VA: Veterans Affairs. \#: $\beta$-agonist, inhaled corticosteroid, tiotropium or ipratropium; ๆ: defined by use of supplemental oxygen, systemic glucocorticoids or antibiotic therapy, or presentation to the emergency department or hospitalisation. 
patient cohort data from the Taiwan National Health Insurance Research Database found that new LABA use was associated with an increased risk of a severe CV event within 30 days of therapy (OR 1.50, $\mathrm{p}<0.001$ compared with non-use of LABA or long-acting muscarinic antagonists (LAMAs)) [123]. However, there was no increase in risk with prevalent (long-term) use. Furthermore, in the SUMMIT (Study to Understand Mortality and Morbidity in COPD) RCT, which was conducted among patients with heightened CV risk, there was no excess of cardiac disorders or arrhythmias among patients treated with the LABA vilanterol compared with a placebo [92]. Observational data regarding new use of short-acting $\beta_{2}$-agonists (SABAs) are also mixed and, in one Canadian database analysis, SABAs did not increase fatal or non-fatal acute myocardial infarction (MI) [71].

There are also data to suggest that $\beta_{2}$-agonists can produce effects that may have a positive impact on CV risk. For example, LABAs provide significant improvements in inspiratory capacity (IC), a marker of lung hyperinflation, versus placebo [128-130]. Lung deflation and improvements in QT kinetics and microvascular oxygen delivery have also been observed [81], while improvements in dyspnoea with indacaterol were associated with improvements in indices of right-ventricular compliance [73]. Inhaled LABAs may also have direct beneficial effects on pulmonary haemodynamics (e.g. pulmonary arterial pressure (PAP)) [27] and reduce the rate of COPD exacerbations [70, 131, 132], which are associated with an increased CV risk and mortality [12].

\section{Muscarinic antagonists}

Similar to LABAs, LAMAs have been associated with both positive and negative CV effects. Anti-muscarinic agents may suppress parasympathetic control of heart rate, which could increase the risk of tachyarrythmias [133]. However, there are various potential mechanisms whereby muscarinic antagonists could lower CV risk, such as by reducing lung hyperinflation [134, 135].

The LAMA tiotropium was shown to reduce lung hyperinflation and improve CV responses to exercise (such as reducing heart rate and blood pressure (BP)) [75]. The reduction in heart rate correlated with an increase in inspiratory reserve volume (IRV) [75], consistent with an observed improvement in CV effects due to mechanical unloading of ventilator muscles. Tiotropium also reduces arterial stiffness [80]. Other potential CV benefits of LAMAs include reduction in exacerbations and an improvement in left-ventricular diastolic function, although not left-ventricular ejection fraction (LVEF) [79].

There are inconsistent results regarding the effects of muscarinic antagonists on CV risk and mortality in COPD. Meta-analyses and cohort analyses reported that muscarinic antagonists, and tiotropium specifically, are not associated with an increase in CV events, risks, or deaths [65, 77]. In contrast, an analysis of Canadian healthcare databases indicated that new ipratropium use was associated with an elevated rate of cardiac arrhythmia in patients with COPD [82, 83] and another meta-analysis reported that ipratropium and tiotropium increased the risk of the composite endpoint of CV mortality, MI and stroke compared with control therapy [76]. However, it has been suggested that the latter meta-analysis had methodological flaws [136], such as the inclusion of many patients from one study and that many CV deaths occurred in patients who were non-compliant with ipratropium. Tiotropium may also have pro-ischaemic and pro-arrhythmic effects [133]. Further data from Taiwan found that new LAMA use (as well as new LABA use) was associated with a 1.52-fold increase in the risk of a severe CV event within 30 days of therapy initiation compared with non-use of a LABA or a LAMA $(p<0.001)$. It was hypothesised that this effect, observed for both LAMAs and LABAs, may be related to sympathetic over-activation or an increase in inflammatory cytokine levels [123]. However, in the same study, there was no risk associated with long-term use [123] and tiotropium has been associated with a trend towards reduced risk for all-cause, CV and respiratory mortality in RCTs [74, 78, 137].

\section{LABA/LAMA combinations}

The CV risk associated with combining long-acting bronchodilators is largely uncertain. A combination of the short-acting bronchodilators salbutamol and ipratropium reduced lung hyperinflation and provided faster QT kinetics as well as larger improvements in microvascular oxygen delivery versus placebo [81]. Furthermore, in the CLAIM study [86], the LABA/LAMA combination indacaterol/glycopyrronium significantly improved left-ventricular and right-ventricular end diastolic volumes, QT and peak forced expiratory volume in $1 \mathrm{~s}(\mathrm{FEV} 1)$, and induced lung deflation compared with placebo.

Large RCTs have not identified additional CV-associated safety concerns with combining long-acting bronchodilators versus monotherapy alone, although studies were not designed to investigate this outcome $[64,138]$. Real-world, primary care data indicated that the addition of a second long-acting bronchodilator to existing LABA or tiotropium treatment was not associated with an increased risk of acute MI, stroke or arrhythmia after 1 year compared with monotherapy, but the risk of developing HF was significantly elevated [66]. Furthermore, HF risk increased by $21 \%$ when patients with prior HF were removed from the 
analysis [66]. The reasons for this elevation are unknown, and further investigation and monitoring are required. However, when comparing LABA/LAMA and ICS/LABA therapy in a retrospective, observational cohort study, CV events were less frequent with LABA/LAMA treatment, with no significant difference observed in the risk of cerebrovascular events [85].

\section{ICS, ICS/LABA and ICS/LABA/LAMA combinations}

The effects of ICS on systemic inflammation $[41,139,140]$ and cardioprotection $[87,88]$ are unclear. Systemic corticosteroids appear to promote progression of atherogenesis, but may also improve recovery from occlusive vascular events and intravascular injury [141, 142]. Lung deflation with fluticasone furoate/ vilanterol improved cardiac function (right-ventricular, left-ventricular and left atrial volumes) versus placebo [91]. Treatment with ICS/LABA also reduced arterial stiffness to a similar extent as tiotropium in a study of 257 patients with COPD, suggesting that the long-acting bronchodilator component of the combination drives this effect [80]. However, no reduction in aortic pulse wave velocity (aPWV) was observed compared with placebo [90, 93].

A hypothesis-generating post hoc analysis of TORCH (Towards a Revolution in COPD Health) indicated that the ICS/LABA combination salmeterol/fluticasone propionate might have beneficial effects on reducing $\mathrm{CV}$ events in certain subgroups of patients, such as those receiving $\mathrm{CV}$ medications at baseline or with moderate COPD [89]. The SUMMIT study investigated this hypothesis by comparing fluticasone furoate/vilanterol, respective monotherapies, and placebo in patients with moderate COPD and CVD or multiple CV risk factors [92]. Fluticasone furoate/vilanterol did not significantly reduce the risk of mortality versus placebo and there were no significant differences when data were analysed by age, sex, baseline therapy or presence of CVD [92]. In addition, there was no effect on the composite CV endpoint [92]. Why SUMMIT did not reproduce the results of the TORCH post hoc analysis is unclear. This could reflect differences in the efficacy of the treatments used, or other methodological considerations related to RCTs [68], as discussed above.

Data on the CV risks or benefits of ICS or ICS/LABA combinations in observational studies are also mixed, with reports that low-dose ICS $\left(50-200 \mu \mathrm{g} \cdot \mathrm{day}^{-1}\right)$ may be associated with a reduction in the risk of acute MI in patients with COPD [87]. Although observational studies have indicated that ICS have benefits on CV mortality, RCTs have not shown any significant effect on CV death [88]. To date, no studies have been designed to investigate the effect of ICS/LABA/LAMA combination therapies on CV events; however, RCTs comparing ICS/LABA/LAMA with LAMA/LAMA or ICS/LABA combinations have provided no evidence of an excess CV risk with triple therapy [143, 144].

\section{Other COPD treatments}

Phosphodiesterase- 4 inhibitors, such as roflumilast, are recommended as an additional therapy on top of triple therapy (a combination of ICS, LABA and LAMA) in inadequately controlled patients in Global Initiative for Chronic Obstructive Lung Disease (GOLD) Group D [1]. Phosphodiesterase inhibitors have anti-inflammatory effects, can reduce the risk of exacerbations [145] and may therefore have beneficial effects on CV events in patients with COPD and increased CV risk. Available data are limited, however, a lower rate of major CV adverse events (AEs) with roflumilast versus placebo has been reported [96]. A more recent meta-analysis did not identify any particular CV safety signal with roflumilast, but it did report an increased incidence of some (non-CV) AEs, which led the authors to conclude that further, long-term safety studies were needed [146].

Theophylline, a further possible treatment for COPD, [1] has anti-inflammatory effects [147] and intravenous theophylline reduces pulmonary vascular resistance (PVR) and improves ventricular function [148]. However, high doses of theophylline have been linked to changes in cardiac electrophysiology [149]. Weak associations of theophylline and arrhythmias, AF and supraventricular tachycardias have been suggested by a UK case-control study [95], and a cohort study of 12301 subjects from Canada indicated that the rate of CV death was greater in users of theophylline (with most deaths occurring in individuals with pre-existing conditions) [94]. While this latter analysis was not specific to patients with COPD, the balance of available data suggests that theophylline should be used with caution in patients with COPD and CVD.

Aside from pharmacological strategies, pulmonary rehabilitation (PR) is recommended for patients with COPD as part of integrated patient management [1]. For those with CVD, it is important to consider both diseases when creating PR programmes [150]. Retrospective analyses and prospective studies provide conflicting insight into the effect of CVD on PR efficacy, with the former suggesting a reduction in the ability to achieve a clinically-important difference in 6-minute walking distance and health status in patients with metabolic diseases versus those without [151]. However, when studied prospectively, this effect was not observed [152]. Furthermore, the effect of PR on CVD risk is largely unknown. A systematic review of studies investigating arterial stiffness in response to PR or an exercise-training programme in 
COPD patients only found three eligible studies, with conflicting results [153]; however, there may be subpopulations of patients who benefit from PR with regards to CV risk [153].

Finally, as an intervention, smoking cessation has the greatest potential to influence the natural history of COPD [1]. A network meta-analysis of pharmacological and behavioural smoking cessation interventions in CVD patients indicated that varenicline and bupropion were associated with greater abstinence than placebo, with increased efficacy also noted with telephone therapy and individual counselling versus usual care [154]. However, a further network meta-analysis found no association between cessation medications and major CV events, although nicotine replacement therapy was associated with an increase in CV events overall (driven predominantly by less serious events) compared with smoking cessation advice alone [155, 156].

In summary, the clinical data suggests that inhaled therapies used in the treatment of COPD are not associated with significant CV risk while smoking cessation remains a core strategy for both COPD and CVD, reducing the overall risk of premature mortality from smoking-related diseases. However, more data are required to establish the long-term safety of COPD pharmacotherapies, particularly among patients at high risk of $\mathrm{CV}$ events who are often excluded from COPD clinical trials. Observational studies suggest that new users of LABAs and LAMAs with COPD are at higher risk of CV events compared with non-use of LABAs and LAMAs, which indicates the need to monitor patients with CV comorbidities, particularly when bronchodilator therapies are initiated.

\section{Effects of CV treatments on COPD $\beta$-blockers}

The European Society of Cardiology (ESC) advocates the use of $\beta$-blockers in HF patients irrespective of the presence of COPD [52], but advises caution in patients with stable coronary artery disease (CAD) and concurrent COPD [157]. The use of $\beta$-blockers in patients with COPD and concurrent CVD has historically been avoided because of concerns about potential adverse pulmonary effects. Bronchial smooth muscle contains adrenergic receptors, primarily of the $\beta_{2}$-subtype, and activation of these receptors by agonists causes bronchodilation [158]. Consequently $\beta$-blockers are contraindicated in patients with COPD due to concerns about the potential for acute bronchospasm [159]. Indeed, the non-selective blockade of $\beta$-receptors by agents such as propranolol has been shown to inhibit the bronchodilator response to $\beta_{2}$-agonists in patients with COPD [160]. However, concerns over the safety of $\beta$-blocker therapy in patients with COPD have resulted in sub-optimal therapy in patients with CVD and comorbid COPD [161, 162].

Data which address these safety concerns are increasing. Selective $\beta_{1}$-blockers (e.g. atenolol, bisoprolol and metoprolol) have a 20 -fold greater affinity for $\beta_{1}$-receptors versus $\beta_{2}$-receptors; thus, this subclass of agents are significantly less likely to induce bronchoconstriction. Clinical trials and large meta-analyses have shown that single-dose and long-term use of selective $\beta_{1}$-blockers does not have a significant effect on FEV1, $\beta$-agonist response, respiratory symptoms, or overall patient condition compared with patients with COPD not receiving $\beta_{1}$-blockers [163-165]. Indeed, selective $\beta_{1}$-blockers and $\beta_{2}$-agonists may have complementary effects, as the use of $\beta_{1}$-blockers in patients with COPD can sensitise $\beta_{2}$-receptors to $\beta_{2}$-agonists [166]. Clinical hesitance to administer selective $\beta_{1}$-blockers when respiratory conditions are present is reflected in review articles and practice guidelines; however, a Cochrane review found that $\beta_{1}$-blockers in mild-to-moderate reversible airway disease or COPD did not produce adverse respiratory effects [163]. Furthermore, selective $\beta_{1}$-blockers do not increase the risk for moderate or severe exacerbations in patients with asthma [167]. This finding is particularly noteworthy given that airflow obstruction reversibility (following $\beta_{2}$-agonist inhalation) is characteristically greater in asthma than in COPD. Thus, one might expect that patients with asthma would be more vulnerable to the adverse effects of $\beta$-blockade than patients with COPD; however, with selective $\beta_{1}$-blockade this appears not to be the case.

Evidence from observational studies suggests that $\beta$-blockers are associated with various benefits in patients with COPD with or without CVD, such as reductions in mortality, hospital admissions, emergency room (ER) visits and COPD exacerbations [97-99, 101-103], although these studies may have been affected by biases from immortal and immeasurable time [168-170].

Furthermore, the continued use of $\beta$-blockers in COPD patients hospitalised for exacerbation did not result in an increase in in-hospital mortality, 30-day readmission or late mechanical ventilation [100]. However, the use of $\beta$-blockers may have little effect on lung function and dynamic hyperinflation [104]. Such data must be interpreted cautiously in the absence of RCT data, particularly given the recent discrepancy between observational and RCT data in CV therapy for COPD discussed below in relation to statins.

\section{Renin-angiotensin-aldosterone system inhibitors ACEls and ARBs}

The renin-angiotensin-aldosterone system (RAAS) has been implicated in various processes in the lungs that may be important in the pathogenesis of COPD, including the induction of pro-inflammatory 
modulators, the generation of reactive oxygen species and the development of pulmonary fibrosis [171]. Data on the effects of RAAS inhibitors in patients with COPD are limited, although a couple of small studies have indicated a potential benefit on pulmonary function and haemodynamics [105, 106]. More recently, an analysis of the Multi Ethnic Study of Atherosclerosis (MESA), including individuals in the general population aged 45-84 years who had no clinical evidence of CV disease, found that baseline use of an ACEI or ARB protected against the progression of emphysema, especially when prescribed at high doses [124]. The authors attributed the effects of such RAAS inhibitors to inhibition of transforming growth factor- $\beta$ signalling in the lung, thereby reducing the progression of airspace enlargement.

Unfortunately, the most common side effect of therapy with ACEIs is cough, which develops in 5-20\% of patients and may be problematic for patients with COPD. Although uncommon, worsening airflow obstruction has been associated with ACEI treatment, leading to suggestions that these agents be used with caution or as a second-line in patients with COPD [158]. No such safety concerns have been reported with ARBs [158]; indeed, data from an observational cohort study among patients with COPD who used ACEIs or ARBs found that ARBs were associated with fewer COPD complications, including severe exacerbations, pneumonia and mortality, than ACEIs [122]. Although these findings require further confirmation, this may suggest that ARBs are a better choice for patients with COPD requiring treatment with a RAAS inhibitor compared with ACEIs [122].

\section{Statins}

Given the high prevalence of CVD and CV risk factors in COPD [3], many patients with COPD receive statin therapy for the primary or secondary prevention of CVD. The lipid-lowering effects of statins are well documented; however, anti-inflammatory effects have also been observed in the airways and CV tissue [172-176] and therefore these agents could have beneficial effects in COPD. Indeed, statin therapy has been shown to improve Borg dyspnoea scores versus placebo in patients with COPD and PH [109] and data from retrospective analyses and prospective observational studies support a role for statins in patients with COPD in terms of reductions in exacerbations, hospitalisations and mortality after an exacerbation $[107,110-112,114,117,177,178]$. However, many of these studies may have been affected by biases from immortal and immeasurable time [179].

In the Rotterdam study, long-term statin use had a beneficial effect on mortality in patients with COPD compared with never use [115]. Observational studies suggest that statins may also be associated with a reduced prevalence of airflow limitation [113] and a reduction in FEV1 decline [108]. However, an analysis of patients with chronic HF and history of COPD found no favourable effect between statins and all-cause mortality, CV death, non-CV death or all-cause hospitalisation [118], Indeed, in the STATCOPE study (a randomised placebo-controlled trial of simvastatin in the prevention of COPD exacerbations), statin therapy did not decrease exacerbation rates [116]. However, patients with diabetes and coronary heart disease were not included in this study and therefore a benefit of statin therapy on patients at CV risk cannot be excluded.

\section{Anticoagulants}

Anticoagulants (e.g. warfarin and newer agents such as apixaban, dabigatran, edoxaban and rivaroxaban) are often used to prevent future thrombotic events in patients with CVD. It is well known that smoking increases the risk of venous thromboembolism (VTE) [180], but patients with severe COPD are also at increased risk of secondary VTE and mortality is higher in patients who have COPD and VTE versus COPD alone [181]. Furthermore, a recent meta-analysis revealed that pulmonary embolism was present in approximately one-sixth of patients who had an acute COPD exacerbation of unknown cause and that these emboli were often in regions indicated for anticoagulant therapy [182]. Thus, use of anticoagulants in at-risk COPD patients may help to reduce future thrombotic-related morbidity and mortality.

\section{Antiplatelet therapy}

Thrombocytosis has been reported in an observational cohort of 1343 patients hospitalised for an acute exacerbation of COPD and antiplatelet therapy correlated with lower 1-year mortality in this study [120]. A national prospective multicentre study also suggests a positive effect of antiplatelet therapy on mortality in patients with COPD [119].

\section{Other CV medications}

Data are limited for the effects of other CV medications in patients with COPD. There are no safety concerns or contraindications regarding the use of calcium channel blockers (CCBs) or aldosterone receptor blockers, such as spironolactone, in patients with COPD [158]. In patients with COPD and hypertension, the use of a thiazide diuretic in combination therapy did not affect the risk of COPD exacerbations [121]. However, respiratory acidosis, a common condition in patients with COPD, may be 
further complicated by the effects of diuretics on electrolyte levels and acid-base balance, leading to development of mixed acid-base disorders [183]. For example, treating patients with COPD and HF with high-dose diuretics can result in metabolic acidosis and metabolic alkalosis, in addition to pre-existing respiratory acidosis [183]. Patients with HF with reduced ejection fraction may also be treated with sacubitril/valsartan, a first-in-class angiotensin receptor-neprilysin inhibitor (ARNI) [184]. However, the potential impact of ARNIs on COPD outcomes has not been studied.

Overall, the evidence to support the use of CV therapies in patients with COPD is reassuring. Indeed, there are some data to suggest that RAAS inhibitors and statins have a protective effect on the progression of COPD although outcomes studies specifically designed to assess the effect of therapy on patients with COPD and CV would be welcome. Furthermore, historic concerns about the safety of $\beta$-blockers are not borne out by the data, which indicates that this class is beneficial in patients with COPD.

\section{Recognising and managing comorbid COPD and CVD}

Comorbidity in COPD is common and can be fatal. Therefore, in order to treat patients appropriately, physicians should proactively search for prevalent and clinically important comorbidities such as CVD. The three most commonly occurring cardiac comorbidities of COPD are AF, HF and IHD [185]. However, non-specific symptoms such as dyspnoea and fatigue are common to all four diseases and acute exacerbations of each of these conditions can result in exacerbation of respiratory symptoms. Indeed, acute respiratory symptoms invariably have mixed pulmonary and cardiac origin [185]. Diagnosing and managing comorbid COPD and CVD and their exacerbations thus remain challenging [186].

\section{Recognition}

Given the impact of CVD in COPD, it is important to recognise and treat CVD and CV risk factors (such as smoking, cholesterol and BP) as early as possible, but practical guidance is limited. Numerous guidelines have been published to assist in the differential diagnosis of COPD and CVD in isolation, but few exist in the setting of comorbidity or multimorbidity. However, Roversi et al. [185] have made important progress in this regard, publishing a set of diagnostic and screening procedures to help differentiate COPD from AF, HF and IHD.

When used in addition to lung function tests, CV risk scores significantly improve the prediction of CV events and mortality in patients with COPD [187]. Over and above guidelines-recommended diagnostic procedures for CVD, further subclinical markers and biomarkers may also assist the assessment and diagnosis of patients in clinical practice. For example, arterial stiffness (as measured by aPWV) has been shown to be predictive of $\mathrm{CV}$ events independent of classic CV factors and has been proposed as a surrogate marker of severity [38]. In addition, a prospective cohort study indicated that angiopoietin-like protein 4 was independently associated with CV function in patients with COPD [188].

There is also potential for pulmonologists to learn from other specialities that recognise the importance of identifying and managing key comorbidities. For example, diabetes guidelines from the American Diabetes Association recognise the need to look beyond blood glucose, highlighting the importance of effectively managing high BP in patients with diabetes [189].

\section{Management}

Key international, regional and local guidelines provide limited recommendations on how to manage patients with COPD and CVD [1, 190-192]. The GOLD strategy document states that the presence of comorbidities should not, in general, alter COPD treatment and that comorbidities should be treated as per usual standards, irrespective of the presence of COPD [1].

In approaching the treatment of COPD in HF patients, the ESC advocate the use of $\beta$-blockers without contraindication for COPD [52]. Unfortunately, despite no consistent evidence contraindicating concurrent $\beta$-blocker and LABA administration, some patients with COPD and CVD are not receiving guideline-based therapies due to historical concerns $[63,193]$ and patients with HF are less likely to receive $\beta$-blockers if they also have COPD $[63,193]$.

In 2016, the National Institute for Health and Care Excellence published a set of clinical guidelines for the assessment, prioritisation and management of patients with commonly occurring multimorbidities [7]. However, more extensive, integrated recommendations specifically concerning CV risk assessment and management in patients with COPD are necessary to optimise management of comorbid COPD and CVD. Indeed, in order to ascertain the most appropriate treatment in patients with further exacerbations despite treatment with a LABA/LAMA, recent proposals for alternative treatment algorithms advocate the assessment of comorbidities [186]. Adoption of strategies aimed at improving outcomes in these 
"cardiopulmonary" patients (e.g. via joint management by respiratory and cardiac health professionals in a cardiopulmonary outpatient clinic) is to be encouraged [185].

\section{Our recommendations}

$\mathrm{CV}$ diseases are often underdiagnosed and under-treated in patients with COPD [18]. Furthermore, treatment with therapies targeted to treat comorbidities such as HF, IHD, AF and hypertension have the potential to modify the natural history of patients with COPD $[115,124]$. We therefore support the active detection and management of comorbidities in COPD and suggest using relevant treatment guidelines for patients without COPD in the absence of more specific information. Our recommendations for clinicians regarding management of patients with both COPD and CVD in clinical practice are as follows: 1) multimorbidity is often overlooked during the diagnosis of an initial (i.e. index) chronic disease; however, all patients who have COPD, CVD or another index chronic disease should be evaluated thoroughly to rule out the presence of additional chronic diseases. This is especially important for index diseases such as COPD, in which treatment is purely symptomatic and does not address underlying pathophysiological causes; 2) consider and measure CV risk in every patient with COPD using a validated score (e.g. QRISK3 [194]).Where this gives intermediate results, consider an individualised assessment of risk, such as coronary calcium score or pulse wave velocity. Assess diffusion capacity for the differential diagnosis of dyspnoea in COPD; 3 ) in patients with COPD, manage comorbid CVD and CV risks according to guidelines. Prescribe $\beta$-blockers only in accordance with approved indications, use cardioselective $\beta_{1}$-blockers (e.g. atenolol, bisoprolol and metoprolol), initiate treatment at the lowest dose and up-titrate slowly. Engage/interact with other medical disciplines to address treating patients with multimorbidity; 4) in patients with COPD, monitor lung hyperinflation routinely via IC assessments; 5) reassess risk and control in response to changes in intervention

\section{Conclusion}

COPD and CVD are complex disorders that frequently co-exist and are associated with worse outcomes than either condition alone. Potential mechanisms have been discussed whereby COPD and CVD may interact and treatments for COPD may help to reduce the risk of CVD. These include mechanical offloading of the CV system through reductions in lung hyperinflation, anti-inflammatory effects and the prevention of exacerbations. Current guidelines offer only limited recommendations for the management of CVD in patients with COPD and there is an urgent need for more extensive and specific recommendations to guide physicians in clinical practice.

Acknowledgements: The authors were assisted in the preparation of the manuscript by Graham Allcock and Elizabeth Andrew (professional medical writers, CircleScience (an Ashfield company, part of UDG Healthcare plc), Tytherington, UK) and Laura Elson (a professional medical writer contracted to CircleScience). Medical writing support was funded by Novartis Pharma AG (Basel, Switzerland).

Conflict of interest: K.F. Rabe reports personal fees (consultant and speakers fees) from AstraZeneca, Boehringer Ingelheim, Novartis, Sanofi, Teva, Intermune, Chiesi Pharmaceuticals and Berlin Chemie, as well as grants from the Ministry of Education and Science, Germany, outside the submitted work. J. Hurst reports grants, personal fees and non-financial support from pharmaceutical companies that make medicines to treat COPD, outside the submitted work. S. Suissa reports grants and personal fees (board membership and research grant) from Novartis and Boehringer Ingelheim, and personal fees (lectures) from AstraZeneca, outside the submitted work.

Support statement: This study was funded by Novartis. Funding information for this article has been deposited with the Crossref Funder Registry.

\section{References}

1 Global Initiative for Chronic Obstructive Lung Disease (GOLD). Global strategy for the diagnosis, management, and prevention of chronic obstructive pulmonary disease (2018 report). https://goldcopd.org/wp-content/uploads/ 2017/11/GOLD-2018-v6.0-FINAL-revised-20-Nov_WMS.pdf Date last accessed: November 28, 2017.

2 Divo M, Cote C, de Torres JP, et al. Comorbidities and risk of mortality in patients with chronic obstructive pulmonary disease. Am J Respir Crit Care Med 2012; 186: 155-161.

3 Smith MC, Wrobel JP. Epidemiology and clinical impact of major comorbidities in patients with COPD. Int J Chron Obstruct Pulmon Dis 2014; 9: 871-888.

4 van den Akker M, Buntinx F, Knottnerus JA. Comorbidity or multimorbidity: what's in a name? A review of literature. Eur J Gen Pract 1996; 2: 65-70.

5 Bhatt SP, Dransfield MT. AECOPD: acute exacerbations of chronic obstructive cardiopulmonary disease? Am J Respir Crit Care Med 2013; 188: 1046-1048.

6 Ording AG, Sorensen HT. Concepts of comorbidities, multiple morbidities, complications, and their clinical epidemiologic analogs. Clin Epidemiol 2013; 5: 199-203.

7 National Guideline Centre. Multimorbidity: clinical assessment and management: assessment, prioritisation and management of care for people with commonly occurring multimorbidity (NICE guideline NG56). London, National Institute for Health and Care Excellence, 2016. www.nice.org.uk/guidance/ng56/evidence/ full-guideline-pdf-2615543103 Date last updated: December 06, 2016. 
Miller J, Edwards LD, Agusti A, et al. Comorbidity, systemic inflammation and outcomes in the ECLIPSE cohort. Respir Med 2013; 107: 1376-1384.

Mannino DM, Thorn D, Swensen A, et al. Prevalence and outcomes of diabetes, hypertension and cardiovascular disease in COPD. Eur Respir J 2008; 32: 962-969.

Westerik JA, Metting EI, van Boven JF, et al. Associations between chronic comorbidity and exacerbation risk in primary care patients with COPD. Respir Res 2017; 18: 31.

Lahousse L, Tiemeier H, Ikram MA, et al. Chronic obstructive pulmonary disease and cerebrovascular disease: a comprehensive review. Respir Med 2015; 109: 1371-1380.

Donaldson GC, Hurst JR, Smith CJ, et al. Increased risk of myocardial infarction and stroke following exacerbation of COPD. Chest 2010; 137: 1091-1097.

Speizer FE, Fay ME, Dockery DW, et al. Chronic obstructive pulmonary disease mortality in six U.S. cities. Am Rev Respir Dis 1989; 140: S49-S55.

Corrao S, Brunori G, Lupo U, et al. Effectiveness and safety of concurrent beta-blockers and inhaled bronchodilators in COPD with cardiovascular comorbidities. Eur Respir Rev 2017; 26: 160123.

Lahousse L, Verhamme KM, Stricker BH, et al. Cardiac
pulmonary disease. Lancet Respir Med 2016; 4: 149-164.

Brook RD, Anderson JA, Calverley PM, et al. Cardiovascular outcomes with an inhaled beta2-agonist/ corticosteroid in patients with COPD at high cardiovascular risk. Heart 2017; 103: 1536-1542.

Cazzola M, Calzetta L, Rinaldi B, et al. Management of chronic obstructive pulmonary disease in patients with cardiovascular diseases. Drugs 2017; 77: 721-732.

Morgan AD, Zakeri R, Quint JK. Defining the relationship between COPD and CVD: what are the implications for clinical practice? Ther Adv Respir Dis 2018; 12: https://doi.org/10.1177/1753465817750524

Maclay JD, MacNee W. Cardiovascular disease in COPD: mechanisms. Chest 2013; 143: 798-807.

Ghoorah K, De Soyza A, Kunadian V. Increased cardiovascular risk in patients with chronic obstructive pulmonary disease and the potential mechanisms linking the two conditions: a review. Cardiol Rev 2013; 21: 196-202.

Rossi A, Aisanov Z, Avdeev S, et al. Mechanisms, assessment and therapeutic implications of lung hyperinflation in COPD. Respir Med 2015; 109: 785-802.

O'Donnell DE, Webb KA, Neder JA. Lung hyperinflation in COPD: applying physiology to clinical practice. COPD Research and Practice 2015; 1: 4.

Parshall MB, Schwartzstein RM, Adams L, et al. An official American Thoracic Society statement: update on the mechanisms, assessment, and management of dyspnea. Am J Respir Crit Care Med 2012; 185: 435-452.

Rennard SI. Clinical approach to patients with chronic obstructive pulmonary disease and cardiovascular disease. Proc Am Thorac Soc 2005; 2: 94-100.

Watz H, Waschki B, Meyer T, et al. Decreasing cardiac chamber sizes and associated heart dysfunction in COPD: role of hyperinflation. Chest 2010; 138: 32-38.

Barr RG, Bluemke DA, Ahmed FS, et al. Percent emphysema, airflow obstruction, and impaired left ventricular filling. N Engl J Med 2010; 362: 217-227.

Cazzola M, Mantero A, Santus P, et al. Doppler echocardiographic assessment of the effects of inhaled long-acting $\beta_{2}$-agonists on pulmonary artery pressure in COPD patients. Pulm Pharmacol Ther 2007; 20: 258-264.

Smith BM, Kawut SM, Bluemke DA, et al. Pulmonary hyperinflation and left ventricular mass: the Multi-Ethnic Study of Atherosclerosis COPD Study. Circulation 2013; 127: 1503-1506.

Smith BM, Prince MR, Hoffman EA, et al. Impaired left ventricular filling in COPD and emphysema: is it the heart or the lungs? The Multi-Ethnic Study of Atherosclerosis COPD Study. Chest 2013; 144: 1143-1151. Ferguson GT. Why does the lung hyperinflate? Proc Am Thorac Soc 2006; 3: 176-179.

Kent BD, Mitchell PD, McNicholas WT. Hypoxemia in patients with COPD: cause, effects, and disease progression. Int J Chron Obstruct Pulmon Dis 2011; 6: 199-208.

Zangiabadi A, De Pasquale CG, Sajkov D. Pulmonary hypertension and right heart dysfunction in chronic lung disease. Biomed Res Int 2014; 2014: 739674.

Boussuges A, Pinet C, Molenat F, et al. Left atrial and ventricular filling in chronic obstructive pulmonary disease. An echocardiographic and Doppler study. Am J Respir Crit Care Med 2000; 162: 670-675.

Louie EK, Rich S, Levitsky S, et al. Doppler echocardiographic demonstration of the differential effects of right ventricular pressure and volume overload on left ventricular geometry and filling. J Am Coll Cardiol 1992; 19: 84-90.

Sievi NA, Clarenbach CF, Camen G, et al. High prevalence of altered cardiac repolarization in patients with COPD. BMC Pulm Med 2014; 14: 55.

Drakopoulou M, Toutouzas K, Michelongona A, et al. Vulnerable plaque and inflammation: potential clinical strategies. Curr Pharm Des 2011; 17: 4190-4209.

Linden F, Domschke G, Erbel C, et al. Inflammatory therapeutic targets in coronary atherosclerosis-from molecular biology to clinical application. Front Physiol 2014; 5: 455.

Vivodtzev I, Tamisier R, Baguet JP, et al. Arterial stiffness in COPD. Chest 2014; 145: 861-875.

Wouters EF. Local and systemic inflammation in chronic obstructive pulmonary disease. Proc Am Thorac Soc 2005; $2: 26-33$.

Sin DD, Man SF. Why are patients with chronic obstructive pulmonary disease at increased risk of cardiovascular diseases? The potential role of systemic inflammation in chronic obstructive pulmonary disease. Circulation 2003; 107: 1514-1519.

1 Sin DD, Man SF, Marciniuk DD, et al. The effects of fluticasone with or without salmeterol on systemic biomarkers of inflammation in chronic obstructive pulmonary disease. Am J Respir Crit Care Med 2008; 177: 1207-1214.

Williams MC, Murchison JT, Edwards LD, et al. Coronary artery calcification is increased in patients with COPD and associated with increased morbidity and mortality. Thorax 2014; 69: 718-723.

Barnes PJ, Celli BR. Systemic manifestations and comorbidities of COPD. Eur Respir J 2009; 33: 1165-1185. 
Kunisaki KM, Dransfield MT, Anderson JA, et al. Exacerbations of chronic obstructive pulmonary disease and cardiac events: a cohort analysis. Am J Respir Crit Care Med 2018; 198: 51-557.

Liu Y, Liu X, Lin G, et al. Decreased CD34+ cell number is correlated with cardiac dysfunction in patients with acute exacerbation of COPD. Heart Lung Circ 2014; 23: 875-882.

Kersul AL, Iglesias A, Rios A, et al. Molecular mechanisms of inflammation during exacerbations of chronic obstructive pulmonary disease. Arch Bronconeumol 2011; 47: 176-183.

Maclay JD, McAllister DA, Johnston S, et al. Increased platelet activation in patients with stable and acute exacerbation of COPD. Thorax 2011; 66: 769-774.

Mills NL, Miller JJ, Anand A, et al. Increased arterial stiffness in patients with chronic obstructive pulmonary disease: a mechanism for increased cardiovascular risk. Thorax 2008; 63: 306-311.

Patel AR, Kowlessar BS, Donaldson GC, et al. Cardiovascular risk, myocardial injury, and exacerbations of chronic obstructive pulmonary disease. Am J Respir Crit Care Med 2013; 188: 1091-1099.

Ito K, Barnes PJ. COPD as a disease of accelerated lung aging. Chest 2009; 135: 173-180.

Lee JH, Cho MH, Hersh CP, et al. Genetic susceptibility for chronic bronchitis in chronic obstructive pulmonary disease. Respir Res 2014; 15: 113.

Ponikowski P, Voors AA, Anker SD, et al. 2016 ESC Guidelines for the diagnosis and treatment of acute and chronic heart failure. Eur Heart J 2016: 37: 2129-2200.

Poole DC, Richardson RS, Haykowsky MJ, et al. Exercise limitations in heart failure with reduced and preserved ejection fraction. J Appl Physiol 2018; 124: 208-224.

Agostoni P, Cattadori G, Guazzi M, et al. Cardiomegaly as a possible cause of lung dysfunction in patients with heart failure. Am Heart J 2000; 140: e24.

Agostoni P, Bussotti M, Cattadori G, et al. Gas diffusion and alveolar-capillary unit in chronic heart failure. Eur Heart J 2006; 27: 2538-2543.

Wasserman K, Zhang YY, Gitt A, et al. Lung function and exercise gas exchange in chronic heart failure. Circulation 1997; 96: 2221-2227.

Patel AR, Donaldson GC, Mackay AJ, et al. The impact of ischemic heart disease on symptoms, health status, and exacerbations in patients with COPD. Chest 2012; 141: 851-857.

$73-83$.

Vestbo J. Systemic inflammation and progression of COPD. Thorax 2007; 62: 469-470.

Yndestad A, Damas JK, Oie E, et al. Systemic inflammation in heart failure-the whys and wherefores. Heart Fai Rev 2006; 11: 83-92.

Kanazawa $\mathrm{H}$, Hirata $\mathrm{K}$, Yoshikawa J. Accelerated decline of lung function in COPD patients with chronic hepatitis C virus infection: a preliminary study based on small numbers of patients. Chest 2003; 123: 596-599. Almagro P, Salvado M, Garcia-Vidal C, et al. Recent improvement in long-term survival after a COPD hospitalisation. Thorax 2010; 65: 298-302.

Fisher KA, Stefan MS, Darling C, et al. Impact of COPD on the mortality and treatment of patients hospitalized with acute decompensated heart failure: the Worcester Heart Failure Study. Chest 2015; 147: 637-645.

Kardos P, Worsley S, Singh D, et al. Randomized controlled trials and real-world observational studies in evaluating cardiovascular safety of inhaled bronchodilator therapy in COPD. Int J Chron Obstruct Pulmon Dis 2016; 11: 2885-2895.

Suissa S, Dell'Aniello S, Ernst P. Long-acting bronchodilator initiation in COPD and the risk of adverse cardiopulmonary events: a population-based comparative safety study. Chest 2017; 151: 60-67.

Suissa S, Dell'Aniello S, Ernst P. Concurrent use of long-acting bronchodilators in COPD and the risk of adverse cardiovascular events. Eur Respir J 2017; 49: 1602245.

Suissa S, Ernst P, Vandemheen KL, et al. Methodological issues in therapeutic trials of COPD. Eur Respir J 2008; 31: 927-933.

Suissa S. Will SUMMIT reach the peak in COPD? Thorax 2014; 69: 405-407.

Suissa S. Run-in bias in randomised trials: the case of COPD medications. Eur Respir J 2017; 49: 1700361.

Calverley PM, Anderson JA, Celli B, et al. Salmeterol and fluticasone propionate and survival in chronic obstructive pulmonary disease. N Engl J Med 2007; 356: 775-789.

Suissa S, Assimes T, Ernst P. Inhaled short acting $\beta$-agonist use in COPD and the risk of acute myocardial infarction. Thorax 2003; 58: 43-46.

Salpeter SR, Ormiston TM, Salpeter EE. Cardiovascular effects of $\beta$-agonists in patients with asthma and COPD: a meta-analysis. Chest 2004; 125: 2309-2321.

Santus P, Radovanovic D, Di Marco S, et al. Effect of indacaterol on lung deflation improves cardiac performance in hyperinflated COPD patients: an interventional, randomized, double-blind clinical trial. Int J Chron Obstruct Pulmon Dis 2015; 10: 1917-1923.

Kesten S, Jara M, Wentworth C, et al. Pooled clinical trial analysis of tiotropium safety. Chest 2006; 130: $1695-1703$.

Travers J, Laveneziana P, Webb KA, et al. Effect of tiotropium bromide on the cardiovascular response to exercise in COPD. Respir Med 2007; 101: 2017-2024.

Singh S, Loke YK, Furberg CD. Inhaled anticholinergics and risk of major adverse cardiovascular events in patients with chronic obstructive pulmonary disease: a systematic review and meta-analysis. JAMA 2008; 300: 1439-1450.

77 Rodrigo GJ, Castro-Rodriguez JA, Nannini LJ, et al. Tiotropium and risk for fatal and nonfatal cardiovascular events in patients with chronic obstructive pulmonary disease: systematic review with meta-analysis. Respir Med 2009; 103: 1421-1429.

78 Celli B, Decramer M, Kesten S, et al. Mortality in the 4-year trial of tiotropium (UPLIFT) in patients with chronic obstructive pulmonary disease. Am J Respir Crit Care Med 2009; 180: 948-955.

79 Nojiri T, Yamamoto K, Maeda $\mathrm{H}$, et al. Effects of inhaled tiotropium on left ventricular diastolic function in chronic obstructive pulmonary disease patients after pulmonary resection. Ann Thorac Cardiovasc Surg 2012; 18: 206-211. 
Pepin JL, Cockcroft JR, Midwinter D, et al. Long-acting bronchodilators and arterial stiffness in patients with COPD: a comparison of fluticasone furoate/vilanterol with tiotropium. Chest 2014; 146: 1521-1530.

Berton DC, Barbosa PB, Takara LS, et al. Bronchodilators accelerate the dynamics of muscle $\mathrm{O}_{2}$ delivery and utilisation during exercise in COPD. Thorax 2010; 65: 588-593.

Wilchesky M, Ernst P, Brophy JM, et al. Bronchodilator use and the risk of arrhythmia in COPD: part 1: Saskatchewan cohort study. Chest 2012; 142: 298-304.

Wilchesky M, Ernst P, Brophy JM, et al. Bronchodilator use and the risk of arrhythmia in COPD: part 2 reassessment in the larger Quebec cohort. Chest 2012; 142: 305-311.

Gershon A, Croxford R, Calzavara A, et al. Cardiovascular safety of inhaled long-acting bronchodilators in individuals with chronic obstructive pulmonary disease. JAMA Intern Med 2013; 173: 1175-1185.

Samp JC, Joo MJ, Schumock GT, et al. Risk of cardiovascular and cerebrovascular events in COPD patients treated with long-acting $\beta_{2}$-agonist combined with a long-acting muscarinic or inhaled corticosteroid. Ann Pharmacother 2017; 51: 945-953.

Hohlfeld JM, Vogel-Claussen J, Biller H, et al. Effect of lung deflation with indacaterol plus glycopyrronium on ventricular filling in patients with hyperinflation and COPD (CLAIM): a double-blind, randomised, crossover, placebo-controlled, single-centre trial. Lancet Respir Med 2018; 6: 368-378.

Huiart L, Ernst P, Ranouil X, et al. Low-dose inhaled corticosteroids and the risk of acute myocardial infarction in COPD. Eur Respir J 2005; 25: 634-639.

Loke YK, Kwok CS, Singh S. Risk of myocardial infarction and cardiovascular death associated with inhaled corticosteroids in COPD. Eur Respir J 2010; 35: 1003-1021.

Calverley PM, Anderson JA, Celli B, et al. Cardiovascular events in patients with COPD: TORCH study results. Thorax 2010; 65: 719-725.

Dransfield MT, Cockcroft JR, Townsend RR, et al. Effect of fluticasone propionate/salmeterol on arterial stiffness in patients with COPD. Respir Med 2011; 105: 1322-1330.

Stone IS, Barnes NC, James WY, et al. Lung deflation and cardiovascular structure and function in chronic obstructive pulmonary disease: a randomized controlled trial. Am J Respir Crit Care Med 2016; 193: 717-726.

Vestbo J, Anderson JA, Brook RD, et al. Fluticasone furoate and vilanterol and survival in chronic obstructive pulmonary disease with heightened cardiovascular risk (SUMMIT): a double-blind randomised controlled trial. Lancet 2016; 387: 1817-1826.

Bhatt SP, Dransfield MT, Cockcroft JR, et al. A randomized trial of once-daily fluticasone furoate/vilanterol or vilanterol versus placebo to determine effects on arterial stiffness in COPD. Int J Chron Obstruct Pulmon Dis 2017; 12: 351-365.

Suissa S, Hemmelgarn B, Blais L, et al. Bronchodilators and acute cardiac death. Am J Respir Crit Care Med 1996; 154: 1598-1602.

Huerta C, Lanes SF, Garcia Rodriguez LA. Respiratory medications and the risk of cardiac arrhythmias. Epidemiology 2005; 16: 360-366.

White WB, Cooke GE, Kowey PR, et al. Cardiovascular safety in patients receiving roflumilast for the treatment of COPD. Chest 2013; 144: 758-765.

Dows SM, Johnson JE, et al. Use of $\beta$ blockers and the risk of death in hospitalised patients with acute exacerbations of COPD. Thorax 2008; 63: 301-305.

Rutten FH, Zuithoff NP, Hak E, et al. $\beta$-blockers may reduce mortality and risk of exacerbations in patients with chronic obstructive pulmonary disease. Arch Intern Med 2010; 170: 880-887.

Short PM, Lipworth SI, Elder DH, et al. Effect of $\beta$ blockers in treatment of chronic obstructive pulmonary disease: a retrospective cohort study. BMJ 2011;342: $\mathrm{d} 2549$.

Stefan MS, Rothberg MB, Priya A, et al. Association between $\beta$-blocker therapy and outcomes in patients hospitalised with acute exacerbations of chronic obstructive lung disease with underlying ischaemic heart disease, heart failure or hypertension. Thorax 2012; 67: 977-984.

Du Q, Sun Y, Ding N, et al. Beta-blockers reduced the risk of mortality and exacerbation in patients with COPD: a meta-analysis of observational studies. PLoS One 2014; 9: e113048.

Puente-Maestu L, Calle M, Ortega-Gonzalez A, et al. Multicentric study on the beta-blocker use and relation with exacerbations in COPD. Respir Med 2014; 108: 737-744.

Bhatt SP, Wells JM, Kinney GL, et al. $\beta$-Blockers are associated with a reduction in COPD exacerbations. Thorax 2016; 71: 8-14.

Key A, Parry M, West MA, et al. Effect of $\beta$-blockade on lung function, exercise performance and dynamic hyperinflation in people with arterial vascular disease with and without COPD. BMJ Open Respir Res 2017; 4: e000164.

Kanazawa H, Hirata K, Yoshikawa J. Effects of captopril administration on pulmonary haemodynamics and tissue oxygenation during exercise in ACE gene subtypes in patients with COPD: a preliminary study. Thorax 2003; 58: 629-631.

6 Andreas S, Herrmann-Lingen C, Raupach T, et al. Angiotensin II blockers in obstructive pulmonary disease: a randomised controlled trial. Eur Respir J 2006; 27: 972-979.

Mancini GB, Etminan M, Zhang B, et al. Reduction of morbidity and mortality by statins, angiotensin-converting enzyme inhibitors, and angiotensin receptor blockers in patients with chronic obstructive pulmonary disease. J Am Coll Cardiol 2006; 47: 2554-2560.

Alexeeff SE, Litonjua AA, Sparrow D, et al. Statin use reduces decline in lung function: VA Normative Aging study. Am J Respir Crit Care Med 2007; 176: 742-747.

Lee TM, Chen CC, Shen HN, et al. Effects of pravastatin on functional capacity in patients with chronic obstructive pulmonary disease and pulmonary hypertension. Clin Sci (Lond) 2009; 116: 497-505.

Mortensen EM, Copeland LA, Pugh MJ, et al. Impact of statins and ACE inhibitors on mortality after COPD exacerbations. Respir Res 2009; 10: 45.

Bartziokas K, Papaioannou AI, Minas M, et al. Statins and outcome after hospitalization for COPD exacerbation: a prospective study. Pulm Pharmacol Ther 2011; 24: 625-631.

Huang CC, Chan WL, Chen YC, et al. Statin use and hospitalization in patients with chronic obstructive pulmonary disease: a nationwide population-based cohort study in Taiwan. Clin Ther 2011; 33: 1365-1370. 
113 Bando M, Miyazawa T, Shinohara $\mathrm{H}$, et al. An epidemiological study of the effects of statin use on airflow limitation in patients with chronic obstructive pulmonary disease. Respirology 2012; 17: 493-498.

114 Wang MT, Lo YW, Tsai CL, et al. Statin use and risk of COPD exacerbation requiring hospitalization. Am J Med 2013; 126: 598-606.

115 Lahousse L, Loth DW, Joos GF, et al. Statins, systemic inflammation and risk of death in COPD: the Rotterdam study. Pulm Pharmacol Ther 2013; 26: 212-217.

116 Criner GJ, Connett JE, Aaron SD, et al. Simvastatin for the prevention of exacerbations in moderate-to-severe COPD. N Engl J Med 2014; 370: 2201-2210.

117 Ingebrigtsen TS, Marott JL, Nordestgaard BG, et al. Statin use and exacerbations in individuals with chronic obstructive pulmonary disease. Thorax 2015; 70: 33-40.

118 Rossi A, Inciardi RM, Rossi A, et al. Prognostic effects of rosuvastatin in patients with co-existing chronic obstructive pulmonary disease and chronic heart failure: a sub-analysis of GISSI-HF trial. Pulm Pharmacol Ther 2017; 44: 16-23.

119 Ekström MP, Hermansson AB, Ström KE. Effects of cardiovascular drugs on mortality in severe chronic obstructive pulmonary disease. Am J Respir Crit Care Med 2013; 187: 715-720.

120 Harrison MT, Short P, Williamson PA, et al. Thrombocytosis is associated with increased short and long term mortality after exacerbation of chronic obstructive pulmonary disease: a role for antiplatelet therapy? Thorax 2014; 69: 609-615.

121 Herrin MA, Feemster LC, Crothers K, et al. Combination antihypertensive therapy among patients with COPD. Chest 2013; 143: 1312-1320.

122 Lai CC, Wang YH, Wang CY, et al. Comparative effects of angiotensin-converting enzyme inhibitors and angiotensin II receptor blockers on the risk of pneumonia and severe exacerbations in patients with COPD. Int $J$ Chron Obstruct Pulmon Dis 2018; 13: 867-874.

123 Wang MT, Liou JT, Lin CW, et al. Association of cardiovascular risk with inhaled long-acting bronchodilators in patients with chronic obstructive pulmonary disease: a nested case-Control Study. JAMA Intern Med 2018; 178: 229-238.

124 Parikh MA, Aaron CP, Hoffman EA, et al. Angiotensin-converting inhibitors and angiotensin II receptor blockers and longitudinal change in percent emphysema on computed tomography. The Multi-Ethnic Study of Atherosclerosis lung study. Ann Am Thorac Soc 2017; 14: 649-658.

$125 \mathrm{Au} \mathrm{DH}$, Lemaitre RN, Curtis JR, et al. The risk of myocardial infarction associated with inhaled $\beta$-adrenoceptor agonists. Am J Respir Crit Care Med 2000; 161: 827-830.

126 Tashkin DP, Celli B, Senn S, et al. A 4-year trial of tiotropium in chronic obstructive pulmonary disease. $N$ Engl J Med 2008; 359: 1543-1554.

127 Iftikhar IH, Imtiaz M, Brett AS, et al. Cardiovascular safety of long acting beta agonist-inhaled corticosteroid combination products in adult patients with asthma: a systematic review. Lung 2014; 192: 47-54.

128 Laforce C, Aumann J, de Teresa Parreño L, et al. Sustained 24-hour efficacy of once daily indacaterol $(300 \mu \mathrm{g})$ in patients with chronic obstructive pulmonary disease: a randomized, crossover study. Pulm Pharmacol Ther 2011; 24: 162-168.

129 Mahler DA, D'Urzo A, Bateman ED, et al. Concurrent use of indacaterol plus tiotropium in patients with COPD provides superior bronchodilation compared with tiotropium alone: a randomised, double-blind comparison Thorax 2012; 67: 781-788.

130 Beier J, Beeh KM, Brookman L, et al. Bronchodilator effects of indacaterol and formoterol in patients with COPD. Pulm Pharmacol Ther 2009; 22: 492-496.

131 Dahl R, Chung KF, Buhl R, et al. Efficacy of a new once-daily long-acting inhaled $\beta_{2}$-agonist indacaterol versus twice-daily formoterol in COPD. Thorax 2010; 65: 473-479.

132 Donohue JF, Fogarty C, Lötvall J, et al. Once-daily bronchodilators for chronic obstructive pulmonary disease: indacaterol versus tiotropium. Am J Respir Crit Care Med 2010; 182: 155-162.

133 Singh S, Loke YK, Enright $\mathrm{P}$, et al. Pro-arrhythmic and pro-ischaemic effects of inhaled anticholinergic medications. Thorax 2013; 68: 114-116.

134 O'Donnell DE, Fluge T, Gerken F, et al. Effects of tiotropium on lung hyperinflation, dyspnoea and exercise tolerance in COPD. Eur Respir J 2004; 23: 832-840.

135 Sanguinetti CM. The lungs need to be deflated: effects of glycopyrronium on lung hyperinflation in COPD patients. Multidiscip Respir Med 2014; 9: 19.

136 Hilleman DE, Malesker MA, Morrow LE, et al. A systematic review of the cardiovascular risk of inhaled anticholinergics in patients with COPD. Int J Chron Obstruct Pulmon Dis 2009; 4: 253-263.

137 Burgel PR, Paillasseur JL, Dusser D, et al. Tiotropium might improve survival in subjects with COPD at high risk of mortality. Respir Res 2014; 15: 64.

138 Calverley PMA, Anzueto AR, Carter K, et al. Tiotropium and olodaterol in the prevention of chronic obstructive pulmonary disease exacerbations (DYNAGITO): a double-blind, randomised, parallel-group, active-controlled trial. Lancet Respir Med 2018; 6: 337-344.

139 Williamson PA, Menzies D, Clearie KL, et al. Dose-response for inhaled fluticasone on airway and systemic inflammation in COPD. Eur Respir J 2011; 37: 206-209.

140 Sin DD, Lacy P, York E, et al. Effects of fluticasone on systemic markers of inflammation in chronic obstructive pulmonary disease. Am J Respir Crit Care Med 2004; 170: 760-765.

141 Walker BR. Glucocorticoids and cardiovascular disease. Eur J Endocrinol 2007; 157: 545-559.

142 Enc Y, Karaca P, Ayoglu U, et al. The acute cardioprotective effect of glucocorticoid in myocardial ischemia-reperfusion injury occurring during cardiopulmonary bypass. Heart Vessels 2006; 21: 152-156.

143 Papi A, Vestbo J, Fabbri L, et al. Extrafine inhaled triple therapy versus dual bronchodilator therapy in chronic obstructive pulmonary disease (TRIBUTE): a double-blind, parallel group, randomised controlled trial. Lancet 2018; 391: 1076-1084.

144 Lipson DA, Barnacle H, Birk R, et al. FULFIL trial: once-daily triple therapy in patients with chronic obstructive pulmonary disease. Am J Respir Crit Care Med 2017; 196: 438-446.

145 Tashkin DP. Roflumilast: the new orally active, selective phophodiesterase-4 inhibitor, for the treatment of COPD. Expert Opin Pharmacother 2014; 15: 85-96. 
Yan JH, Gu WJ, Pan L. Efficacy and safety of roflumilast in patients with stable chronic obstructive pulmonary disease: a meta-analysis. Pulm Pharmacol Ther 2014; 27: 83-89.

Barnes PJ. Theophylline. Am J Respir Crit Care Med 2013; 188: 901-906.

Matthay RA, Mahler DA. Theophylline improves global cardiac function and reduces dyspnea in chronic obstructive lung disease. J Allergy Clin Immunol 1986; 78: 793-799.

Eiriksson CE J, Writer SL, Vestal RE. Theophylline-induced alterations in cardiac electrophysiology in patients with chronic obstructive pulmonary disease. Am Rev Respir Dis 1987; 135: 322-326.

Triest FJ, Singh SJ, Vanfleteren LE. Cardiovascular risk, chronic obstructive pulmonary disease and pulmonary rehabilitation: can we learn from cardiac rehabilitation? Chron Respir Dis 2016; 13: 286-294.

Crisafulli E, Costi S, Luppi F, et al. Role of comorbidities in a cohort of patients with COPD undergoing pulmonary rehabilitation. Thorax 2008; 63: 487-492.

Crisafulli E, Gorgone P, Vagaggini B, et al. Efficacy of standard rehabilitation in COPD outpatients with comorbidities. Eur Respir J 2010; 36: 1042-1048.

Aldabayan YS, Alrajeh AM, Lemson A, et al. Pulmonary rehabilitation and cardiovascular risk in COPD: a systematic review. COPD Research and Practice 2017; 3: 7.

Suissa K, Lariviere J, Eisenberg MJ, et al. Efficacy and safety of smoking cessation interventions in patients with cardiovascular disease: a network meta-analysis of randomized controlled trials. Circ Cardiovasc Qual Outcomes 2017; 10: e002458.

Mills EJ, Thorlund K, Eapen S, et al. Cardiovascular events associated with smoking cessation pharmacotherapies: a network meta-analysis. Circulation 2014; 129: 28-41.

Dollerup J, Vestbo J, Murray-Thomas T, et al. Cardiovascular risks in smokers treated with nicotine replacement therapy: a historical cohort study. Clin Epidemiol 2017; 9: 231-243.

Montalescot G, Sechtem U, Achenbach S, et al. 2013 ESC guidelines on the management of stable coronary artery disease. Eur Heart J 2013; 34: 2949-3003.

Chandy D, Aronow WS, Banach M. Current perspectives on treatment of hypertensive patients with chronic obstructive pulmonary disease. Integr Blood Press Control 2013; 6: 101-109.

Matera MG, Calzetta L, Cazzola M. $\beta$-Adrenoceptor modulation in chronic obstructive pulmonary disease: present and future perspectives. Drugs 2013; 73: 1653-1663.

Chang CL, Mills GD, McLachlan JD, et al. Cardio-selective and non-selective beta-blockers in chronic obstructive pulmonary disease: effects on bronchodilator response and exercise. Intern Med J 2010; 40: 193-200.

Hawkins NM, Wang D, Petrie MC, et al. Baseline characteristics and outcomes of patients with heart failure receiving bronchodilators in the CHARM programme. Eur J Heart Fail 2010; 12: 557-565.

Jabbour A, Macdonald PS, Keogh AM, et al. Differences between beta-blockers in patients with chronic heart failure and chronic obstructive pulmonary disease: a randomized crossover trial. J Am Coll Cardiol 2010; 55: 1780-1787.

Salpeter S, Ormiston T, Salpeter E. Cardioselective beta-blockers for chronic obstructive pulmonary disease. Cochrane Database Syst Rev 2005: 4: CD003566.

Albouaini K, Andron M, Alahmar A, et al. Beta-blockers use in patients with chronic obstructive pulmonary disease and concomitant cardiovascular conditions. Int J Chron Obstruct Pulmon Dis 2007; 2: 535-540.

Kargin F, Takir HB, Salturk C, et al. The safety of beta-blocker use in chronic obstructive pulmonary disease patients with respiratory failure in the intensive care unit. Multidiscip Respir Med 2014; 9: 8.

Salpeter SR. Cardioselective beta blocker use in patients with asthma and chronic obstructive pulmonary disease: an evidence-based approach to standards of care. Cardiovasc Rev Rep 2003; 24: 11.

Morales DR, Lipworth BJ, Donnan PT, et al. Respiratory effect of beta-blockers in people with asthma and cardiovascular disease: population-based nested case control study. BMC Med 2017; 15: 18 .

Lalmohamed A, Stojanovic D, Durand M, et al. Benefits of beta blockers in COPD patients and immortal/ immeasurable time bias BMJ 2011; 342: d2549.

Suissa S, Ernst P. Biases in the observational study of $\beta$ blockers in COPD. Thorax 2008; 63: $1026-1027$.

Suissa S, Ernst P. $\beta$-blockers for COPD inpatients. Thorax 2012; 67: 936-937.

Shrikrishna D, Astin R, Kemp PR, et al. Renin-angiotensin system blockade: a novel therapeutic approach in chronic obstructive pulmonary disease. Clin Sci (Lond) 2012; 123: 487-498.

2 Davis BB, Zeki AA, Bratt JM, et al. Simvastatin inhibits smoke-induced airway epithelial injury: implications for COPD therapy. Eur Respir J 2013; 42: 350-361.

Inoue I, Goto S, Mizotani K, et al. Lipophilic HMG-CoA reductase inhibitor has an anti-inflammatory effect: reduction of MRNA levels for interleukin-1 $\beta$, interleukin-6, cyclooxygenase-2, and p22phox by regulation of peroxisome proliferator-activated receptor alpha (PPARalpha) in primary endothelial cells. Life Sci 2000; 67: $863-876$.

4 Kinlay S, Schwartz GG, Olsson AG, et al. High-dose atorvastatin enhances the decline in inflammatory markers in patients with acute coronary syndromes in the MIRACL study. Circulation 2003; 108: 1560-1566.

Neukamm A, Hoiseth AD, Einvik G, et al. Rosuvastatin treatment in stable chronic obstructive pulmonary disease (RODEO): a randomized controlled trial. J Intern Med 2015; 278: 59-67.

Wang W, Le W, Ahuja R, et al. Inhibition of inflammatory mediators: role of statins in airway inflammation. Otolaryngol Head Neck Surg 2011; 144: 982-987.

Blamoun AI, Batty GN, DeBari VA, et al. Statins may reduce episodes of exacerbation and the requirement for intubation in patients with COPD: evidence from a retrospective cohort study. Int J Clin Pract 2008; 62: 1373-1378.

Soyseth V, Brekke PH, Smith P, et al. Statin use is associated with reduced mortality in COPD. Eur Respir J 2007; 29: 279-283.

Suissa S. Co-morbidity in COPD: the effects of cardiovascular drug therapies. Respiration 2010; 80: 3-7.

Cheng YJ, Liu ZH, Yao FJ, et al. Current and former smoking
a systematic review and meta-analysis. PLoS Med 2013; 10: e1001515. Borvik T, Braekkan SK, Enga K, et al. COPD and risk of venous thromboembolism and mortality in a general population. Eur Respir J 2016; 47: 473-481. 
Aleva FE, Voets L, Simons SO, et al. Prevalence and localization of pulmonary embolism in unexplained acute exacerbations of COPD: a systematic review and meta-analysis. Chest 2017; 151: 544-554.

183 Bruno CM, Valenti M. Acid-base disorders in patients with chronic obstructive pulmonary disease: a pathophysiological review. J Biomed Biotechnol 2012; 2012: 915150.

184 McMurray JJ, Packer M, Desai AS, et al. Angiotensin-neprilysin inhibition versus enalapril in heart failure. N Engl J Med 2014; 371: 993-1004.

185 Roversi S, Fabbri LM, Sin DD, et al. Chronic obstructive pulmonary disease and cardiac diseases. An urgent need for integrated care. Am J Respir Crit Care Med 2016; 194: 1319-1336.

186 Miravitlles M, D’Urzo A, Singh D, et al. Pharmacological strategies to reduce exacerbation risk in COPD: a narrative review. Respir Res 2016; 17: 112.

187 Lee HM, Lee J, Lee K, et al. Relation between COPD severity and global cardiovascular risk in US adults. Chest 2012; 142: 1118-1125.

188 Waschki B, Kirsten AM, Holz O, et al. Angiopoietin-like protein 4 and cardiovascular function in COPD. BMJ Open Respir Res 2016; 3: e000161.

189 Standards of medical care in diabetes - 2014. Diabetes Care 2014; 37: Suppl. 1, S14-S80.

190 Miravitlles M, Soler-Cataluna JJ, Calle M, et al. Spanish guidelines for management of chronic obstructive pulmonary disease (GesEPOC) 2017. Pharmacological treatment of stable phase. Arch Bronconeumol 2017; 53: 324-335.

191 Montes de Oca M, Lopez Varela MV, Acuna A, et al. ALAT-2014 chronic obstructive pulmonary disease (COPD) clinical practice guidelines: questions and answers. Arch Bronconeumol 2015; 51: 403-416.

192 National Clinical Guideline Centre. Management of chronic obstructive pulmonary disease in adults in primary and secondary care (clinical guideline CG101). London, National Clinical Guideline Centre, 2010. www.nice.org. uk/guidance/cg101/evidence/full-guideline-pdf-134519581 Date last updated: June 22, 2010.

193 Tavazzi L, Swedberg K, Komajda M, et al. Clinical profiles and outcomes in patients with chronic heart failure and chronic obstructive pulmonary disease: an efficacy and safety analysis of SHIFT study. Int J Cardiol 2013; 170: $182-188$.

194 Hippisley-Cox J, Coupland C, Brindle P. Development and validation of QRISK3 risk prediction algorithms to estimate future risk of cardiovascular disease: prospective cohort study. BMJ 2017; 357: j2099. 